\title{
Flyover noise measurements of a spiraling noise abatement approach procedure
}

\author{
Lothar Bertsch* and Gertjan Looye ${ }^{\dagger}$ \\ German Aerospace Center (DLR) \\ Eckhard Anton ${ }^{\ddagger}$ \\ ILR RWTH Aachen University \\ Stefan Schwanke ${ }^{\S}$ \\ DFS Deutsche Flugsicherung GmbH
}

\begin{abstract}
DLR is investigating alternative low-noise approach procedures. One such procedure involves approaching the airport at a considerably higher altitude compared with standard landing trajectories, followed by a spiraling descent (helix flight path) shortly before the runway threshold. In this way, high ground noise levels by approaching aircraft are dislocated away from the common approach path and concentrated in the area near the helix path, i. e. in direct vicinity of the airport. Ground noise levels along the entire flight path prior to the helix are significantly reduced. The effectiveness of this procedure, referred to as Helical Noise Abatement Procedure (HeNAP), has been quantified by means of computational simulation analyses. These analyses also focused on aspects such as increased fuel burn and the occurrence of multiple noise events below the helix. In June 2009 a new DLR autopilot especially capable of tracking curved flight path trajectories was flight tested. Three HeNAPs were included in the flight plan, as well as standard and steep landing approaches. In addition, dedicated fly-over noise measurements were organized, supported by RWTH Aachen University. Twelve ground microphones have been installed along the common approach path and the helical flight segment. Despite adverse wind conditions at the only available test day, the measured data confirm the predicted noise dislocation effects. High noise levels have been limited to observer locations around the helix. DLR noise prediction methods have been compared with the experimental data. Predicted trends and noise dislocation effects are in good agreement with the measurements whereas the absolute numerical values show discrepancies. The flight test was closely accompanied by a R\&D member of DFS to study the impact of spiraling procedures on ATM integration and air traffic controller workload, e.g. increased interaction with the pilots. Obviously, a spiraling approach procedure would not be implemented into the existing air traffic scenario with its common approach paths and highly frequented airports. The operational and economic environment still need more detailed investigation. Helical approaches become more feasible for implementation at small, low-frequented regional airports or during night hours to avoid possible noise related curfews.
\end{abstract}

\section{Nomenclature}

$\begin{array}{llll}D & \text { Diameter } & \text { DLR } & \text { German Aerospace Center } \\ \text { AGL } & \text { Above ground level } & \text { EDVE } & \text { ICAO airport code: Braunschweig } \\ \text { ATM } & \text { Air Traffic Management } & \text { HeNAP } & \text { Helical Noise Abatement Procedure } \\ \text { A /P } & \text { Autopilot } & \text { ICAO } & \text { International Civil Aviation Organization } \\ \text { DFS } & \text { Deutsche Flugsicherung GmbH } & \text { ILS } & \text { Instrument Landing System }\end{array}$

\footnotetext{
${ }^{*}$ DLR, Institute of Aerodynamics and Flow Technology, Lilienthalplatz 7, 38108 Braunschweig, Germany

${ }^{\dagger}$ DLR, Institute of Robotics and Mechatronics, Muenchner Strasse 20, 82234 Wessling, Germany

${ }^{\ddagger}$ RWTH Aachen, Institute of Aeronautics and Astronautics, Wuellnerstr. 7, 52062 Aachen, Germany

$\S$ DFS Deutsche Flugsicherung GmbH, Am DFS-Campus 10, 63225 Langen, Germany
} 


\begin{tabular}{|c|c|c|c|}
\hline LNAV & Lateral navigation & $r$ & Radius \\
\hline MicX & Ground microphone at observer location $\mathrm{X}$ & $V_{C A S}$ & Calibrated airspeed \\
\hline$S P L$ & Sound pressure level, $[\mathrm{dB}]$ & $\gamma$ & Inertial flight path angle \\
\hline$S P L(A)$ & A-weighted SPL, $[\mathrm{dBA}]$ & $\sigma$ & Ground resistivity to air, $\left[\frac{k N \cdot s}{4}\right]$ \\
\hline PANAM & Parametric Aircraft Noise Analysis Module & & Inertial track angle $\left[m^{4}\right.$ \\
\hline $\begin{array}{l}\text { RJTT } \\
\text { SHADOW }\end{array}$ & $\begin{array}{l}\text { ICAO airport code: Tokyo Haneda Airport } \\
\text { DLR prediction tool for noise shielding effects }\end{array}$ & Suhscrint & \\
\hline VMC & Visual meteorological conditions & Subscript & \\
\hline VNAV & Vertical navigation & helix & Helical Hight segment \\
\hline WGS & World Geodetic System, here: WGS84 & $\begin{array}{l}\text { init } \\
\text { final }\end{array}$ & $\begin{array}{l}\text { Inital straight segment } \\
\text { Final straight segment }\end{array}$ \\
\hline & Altitude & center & Helix center \\
\hline $\begin{array}{l}n_{\text {turn }} \\
R\end{array}$ & $\begin{array}{l}\text { Number of full turns } \\
\text { GPS position }\end{array}$ & end & End of flight segment \\
\hline
\end{tabular}

\section{Introduction}

The vast increase in commercial air traffic on the one hand and the growing pressure to reduce its environmental impact on the other hand, require radical solutions to achieve considerably lower emission levels of noise and engine emissions. The solutions are sought both at the aircraft level, e. g. by development of more efficient and silent engines, as well as at the operational level, e. g. by developing more environmentalfriendly take-off and approach procedures.

In order to decrease aircraft noise levels in noise sensitive areas, the German Aerospace Center (DLR) has investigated the so-called Helical Noise Abatement Procedure ${ }^{1}$ (HeNAP). This procedure is based on concepts for simultaneous non-interfering operation (SNI) by $\mathrm{Hange}^{2}$ and $\mathrm{Hall}^{3}$. It involves the aircraft approaching the airport at a considerably higher altitude compared to standard ILS approaches and then performing a spiraling descent shortly before the runway threshold. The investigation was performed by means of computer analysis, using a new DLR tool chain for the environmental analysis of aircraft flight trajectories. The process comprises tools for aerodynamics, flight mechanics, engine cycle modeling, and the prediction of aircraft noise and engine emission ${ }^{1}$.

The main conclusions from this study are the following: depending on the initial approach altitude and the geometry of the helix ${ }^{\mathrm{a}}$, the high ground noise levels of approaching aircraft are dislocated away from the common ILS approach path into the area around the spiraling descend. This will significantly reduce ground noise impact along the entire flight path prior to the helix. Yet, at the same time each approaching aircraft causes multiple flyover events in the vicinity of the helix hence increase ground noise pollution in this area. Furthermore, fuel use and flight time increase along the helical approach procedure compared to standard ILS approaches.

In parallel to the computer analysis a flight test was prepared in another DLR project ${ }^{\mathrm{b}}$ to evaluate a newly developed autopilot with improved weather capabilities ${ }^{4}$. To this end, the autopilot was implemented on DLR's Advanced Technologies Testing Aircraft System (ATTAS), see Fig. 1. The intention was to test this autopilot a.o. in the form of a helical inertial flight trajectory at a constant airspeed, since this artificially causes lateral and horizontal wind shears, even in the presence of a little steady wind. The idea arose to perform this test trajectory in the form of a landing approach, resulting in the aforementioned HeNAP trajectory. After it turned out that this was possible from an operational and safety point of view, it was decided to combine the flight test with a ground noise measurement campaign. This would allow to validate the HeNAP results from the computational analysis, in particular to confirm the predicted noise reduction potential.

The HeNAP obviously raises operational question marks, such as air traffic control aspects, which had not been considered so far. For this reason a representative of the German air navigation service provider DFS observed the approaches from the airport tower.

This paper describes the preparation, conducting, and preliminary results of the flight test, with emphasis on the noise abatement aspects. It is structured as follows: In Section II the HeNAP will be described in more detail. In Section III the computational noise analysis of the HeNAP and some illustrative results will be presented. Section IV describes equipment, preparation, and actual execution of the flight test. Section $\mathrm{V}$ discusses initial results. These include the validation of basic principle of the HeNAP, the computational noise analysis and the performance of the autopilot, as well as operational aspects based on crew comments and observations from the airport tower. Finally, conclusions will be discussed in Section VI.

\footnotetext{
${ }^{\mathrm{a}}$ e. g. number of full turns, turn radius, rate of descent

${ }^{\mathrm{b}} \mathrm{DLR}$ internal project Weather and Flying

${ }^{\mathrm{c}}$ DFS Deutsche Flugsicherung GmbH
} 


\section{Spiraling approach procedure}

The HeNAP trajectory and its main characteristic parameters are depicted in Fig.2. The trajectory consists of three segments. The first, initial segment is a straight path, which is easily captured by the LNAV/VNAV autopilot modes and allows the aircraft to be configured for the second, helical segment. Geometrically, the actual helix is initiated at the point where the initial straight segment perpendicularly passes the helix centre. After the helical segment, the trajectory ends with straight approach segment, allowing the aircraft to be brought in trimmed, wings-level flight, from where the autopilot may switch to its landing modes (flare, retard, de-crab) or from where the pilot may take over. This final segment will naturally be identical to the standard runway approach path; its length will be a compromise between safety and its effectiveness for noise abatement (the shorter the better).

The parameters $R_{\text {center }}, r_{\text {helix }}, V_{C A S, h e l i x}$, and $h_{\text {end }}$ may be (slowly) changed during the maneuver. The entry height $h_{\text {init }}$, the final altitude $h_{\text {end }}$, and the number of turns determine the rate of descent. The altitude is barometric (QNH). The flight path and track angles of the final segment are determined by the runway heading and glide slope angle. In Fig.2 the flight path angle of the initial segment $\left(\gamma_{\text {init }}\right)$ is zero. This is not a prerequisite, as will be shown in the simulation studies. The same holds for the initial track angle $\chi_{\text {init }}$. In the figure it is identical to the runway heading, but the spiraling descent may start from any direction.

\section{Computational analysis of the HeNAP}

A new tool chain for the environmental analysis of aircraft flight trajectories has been applied to investigate spiraling approach procedures ${ }^{1}$. Noise shielding effects and the overall ground noise impact are predicted with the new DLR tools SHADOW ${ }^{5}$ and PANAM ${ }^{6}$ respectively. Predicted noise isocontour plots illustrate the concept of spiraling noise abatement approach procedures at standard atmosphere condition with no wind for a conventional transport aircraft ${ }^{\mathrm{d}}$. The max. SPL(A) noise footprint for this aircraft along the reference approach is depicted in Fig. 3(a). Isocontour areas for the investigated helical approaches with 2, 3, and 4 helical flight segments are shown in Fig. 3(b) to 3(d) respectively. Flight path angles were held constant and limited to $-3^{\circ}$. A constant helix radius $r_{\text {helix }}$ of $2000 \mathrm{~m}$ was selected to guarantee passenger comfort by preventing high bank angles and decelerations. To maximize ground noise reduction along the HeNAP, the initial flight segment prior to the helix was simulated identical to the reference approach with a glide slope $\gamma_{\text {init }}$ of $-3^{\circ}$.

A considerable increase in flight time, fuel consumption, and gaseous emissions is predicted for all helical approaches, as presented in Tab. 1. Obviously, a trade-off between economical flight performance and low-noise operation becomes inevitable. Comparing the reference noise isocontour areas with the HeNAP isocontour lines demonstrates the significant noise reduction potential prior to the spiraling flight segments. The highlighted lines in each plot represent selected isocontour areas. High noise levels are dislocated away from the common approach path and concentrated within an area around the ground track of the helical flight segment. Obviously, this limited area will then be subject to multiple flyover events for each approaching aircraft hence increased noise pollution. The isocontour plots show the maximum noise levels that have been predicted for each flyover event.

Ideally, the helix location can be selected in such a way that high noise levels are kept within airport borders or limited to low-populated regions and industrial zones. To further illustrate this, an airport with optimal geographical location was identified without consideration of ATM aspects. A three-segmented HeNAP and a conventional approach ${ }^{\mathrm{e}}$ on runway $34 \mathrm{~L}$ of Tokyo Haneda International Airport (RJTT) have been simulated. The HeNAP was applied at RJTT in order to minimize approach noise impact in the residential areas located southeast of the airport. Fig. 4 shows the predicted noise footprints for both procedures simply copied onto an area map of the Tokyo Bay. The simulation demonstrates a noise reduction potential for areas where air traffic induced community noise plays a major role ${ }^{7}$, i. e. in the area along the common ILS approach path (waypoints $M I C K Y$ and $K O I T O$ ).

\footnotetext{
${ }^{\mathrm{d}}$ here: conventional, medium-range transportation aicraft with under-the-wing engine installation (ATTAS: over-the-wing engine installation)

'Instrument Approach Chart LOC Z RWY34L
} 


\section{Flight test and flyover noise measurements}

\section{A. Test aircraft and autopilot}

The flight test was carried out in the frame of the DLR internal project Weather and Flying. Within this project, the DLR Institute of Robotics and Mechatronics is developing and applying methods for the design of feedback flight control laws with the aim to considerably improve aircraft closed-loop behaviour under adverse atmospheric conditions. The autopilot control laws flown during the described test flight are described in more detail in Ref. ${ }^{4}$. Although it contains most modes of operation of standard autopilots (including autoland), for the test flight its modes for helix tracking and steep landing approaches were of particular interest.

Tracking a helical flight path is a challenging task for an autopilot and very suitable for assessing its over-all behaviour and capabilities. During a helix maneuver, one basically encounters a considerable amount of challenges in autopilot design: coordination of longitudinal and lateral modes, accurate tracking of a flight path that is continuously "bending away", and combined tracking of inertial (the flight path) and air massbased references (the airspeed). From an atmospheric point of view, during a helix maneuver even little constant wind results in continuous wind shears in lateral and longitudinal directions.

For the flight test, the autopilot control laws were implemented on DLRs Advanced Technologies Testing Aircraft System (ATTAS), see Fig. 1. The aircraft is based on a VFW 614 civil transport aircraft equipped with two Rolls-Royce M45 H engines. ATTAS is equipped with a unique in-flight simulation architecture and a fly-by-wire flight control system, allowing for implementation of new flight control laws with low effort. Before flight, the complete flight plan was reviewed in the ATTAS simulator operated by the DLR Institute of Flight Systems. Since the flight control laws were tested in flight for the first time, the initial segment was chosen to be straight and level, i.e. $\gamma_{\text {init }}=0$. Three turns were selected, which is a compromise between time for control law evaluation and available flight time.

\section{B. Flight test plan}

The flight test plan consisted of seven approaches to runway 26 of the Forschungsflughafen Braunschweig (EDVE). The aircraft's configuration along all flights was held constant with gear deployed and flaps on $14^{\circ}$. The reference time (flight time) for overall data correlation was initialized, i. e. flight time set to $0.0 \mathrm{~s}$, along each test flight at a ground distance of $12 \mathrm{~km}$ to the runway threshold.

- flight1: A standard $3^{\circ}$ ILS approach, followed by a go-around when reaching $500 \mathrm{ft}$ AGL.

- flight2: A steep 6-7 $7^{\circ}$ approach, starting at a barometric altitude of $3000 \mathrm{ft}$ QNH. Capturing the ILS from above. Go-around when reaching $500 \mathrm{ft}$ AGL. Objectives were testing of the vertical path and speed modes, and vertical path and glide slope capture logic.

- flight3: Second steep approach.

- flight4: Helical Noise Abatement Procedure, starting at an altitude of $7500 \mathrm{ft}$, ILS capture after the last turn, followed by a go-around when reaching $500 \mathrm{ft}$ AGL. The flight path angle $\gamma_{\text {init }}$ along the initial flight segment was set to zero for simplicity. This maneuver was intended as the all-up test of the autopilot control laws.

- flight5: Second HeNAP.

- flight6: Third HeNAP.

- flight7: Second standard ILS approach, continued into the landing and roll-out (performed manually) marking the end of the test flight.

\section{Procedures}

The helical approach trajectory is depicted schematically in Fig. 2. As already mentioned before, the maneuver is initiated in an altitude $h_{\text {init }}$ of $7500 \mathrm{ft} \mathrm{QNH}$ in the direction of the runway. The altitude is held constant $\left(\gamma_{\text {init }}=0\right)$ until the aircraft passes the helix center, which is located to the right. The helix centre in turn is located at a distance $D_{\text {helix }}$ of $3200 \mathrm{~m}$ before the runway threshold. The latter point is also the reference for computing local $\mathrm{x}, \mathrm{y}, \mathrm{z}$ coordinates. After exactly three full turns with a radius $r_{\text {helix }}$ of $2000 \mathrm{~m}$ the maneuver ends with the final straight trajectory, which matches the standard ILS approach path. Shortly before ending the final circle, the ILS signal is checked. If the aircraft is within the cone, 
the autopilot changes into its ILS tracking mode. Otherwise, flight continues in runway direction, but at constant altitude. The airspeed $V_{C A S, \text { helix }}$ is held constant at $160 \mathrm{kts}(82 \mathrm{~m} / \mathrm{s})$. During the final quarter of the last turn, it is reduced to the agreed final approach speed $V_{C A S, f i n a l}$ of $140 \mathrm{kts}(72 \mathrm{~m} / \mathrm{s})$.

The steep approaches were initiated at an altitude of $3000 \mathrm{ft}$ QNH. The transition from steep into the standard approach path was planned at the same distance $D_{\text {helix }}$ from the runway threshold as the helix centre. The transition from horizontal into the steep approach was fixed to an altitude of $6500 \mathrm{ft}$. In this way, the trajectory is fully defined. An approach speed of $140 \mathrm{kts}(72 \mathrm{~m} / \mathrm{s})$ CAS was selected.

\section{Test set-up}

\section{Noise measurement equipment and data acquisition system}

The flight trajectories and corresponding operational aircraft data were recorded on board of the ATTASaircraft. The trajectory data contained GPS-time for synchronization with measured ground noise data. In addition to official airport weather data, ambient temperature, relative humidity, static air pressure, and wind speed/direction have been obtained every 15 minutes during the flyover campaign using a pole mounted weather sensor system installed close to Mic8, see Fig. 5. The data was measured $10 \mathrm{~m}$ above the ground, according to the aircraft noise certification standards 8 .

Noise data were acquired using an autonomous field noise measurement system povided by the Institute of Aeronautics and Astronautics, RWTH Aachen University. The system consists of plate mounted microphones and a laptop-based data acquisition system conforming to DIN EN 61265. The digitized sound pressure signal was recorded with a sampling rate of $48 \mathrm{kHz}$ and a 16 bit resolution. The measurement system further includes a GPS unit which allows the GPS time and the puls-per-second (PPS) to be recorded along with the sound pressure signal for data synchronization. Furthermore, the GPS position is stored in the noise data header which allows for the precise determination of the geometric relation between aircraft trajectory and microphone position on the ground. A ground board microphone arrangement was chosen originally developed for noise certification of General Aviation aircraft (ICAO Annex 16, Chapter 10). The microphone is mounted eccentrically in an inverted position at $7 \mathrm{~mm}$ above the circular metal ground plate, see Fig. 6 . This setup is used to minimize interference effects in the frequency range from $50 \mathrm{~Hz}$ up to $10 \mathrm{kHz}$ typically used for aircraft noise analysis. The sound wave reflexion on the ground plate results in a sound pressure doubling at the microphone, thus $6 \mathrm{~dB}$ have to be deducted from the presented sound pressure levels in oder to retrieve the free-field levels. Detailed analysis of the interference characteristics of this microphone setup can be found in Ref. ${ }^{9,10}$. Signal processing and calculation of the A-weighted Sound Pressure Level SPL(A) was performed according to the aircraft noise certification standards ${ }^{8}$.

No noise data correction ${ }^{\mathrm{f}}$ was applied because the focus of the campaign was on the one hand to evaluate ground noise impact instead of noise emission at the source and on the other hand to provide uncorrected noise data to validate the prediction codes.

\section{Observer locations}

A total of 12 microphone stations were installed around Forschungsflughafen Braunschweig. Observer locations along the flight ground track of the common approach path and of the HeNAP have been selected. 6 microphones have been installed along the common approach path and the helical flight segments respectively. The coordinates for the ground microphones are given in Tab. 2 and the layout is depicted in Fig. 7 and 8. All flights are approaching from the east towards EDVE runway 26. The observer locations along the common approach path have been selected such that the noise reduction potential of the steep approach and the HeNAP compared to the reference path can be demonstrated. Locations along the helical flight ground track have been selected in order to investigate noise emission along the curved flight segments.

\section{Preliminary analysis of flight test results}

\section{A. Tracking of the flight path}

Fig. 7 depicts one HeNAP, one steep, and one standard approach trajectory out of the seven approaches performed during the flight test. The three HeNAP approaches turned out to be nearly identical. As a result,

\footnotetext{
${ }^{f}$ e.g. de-Dopplerization and correction for atmospheric absorbtion
} 
the projection all HeNAP trajectories on the ground are nearly perfect circles and hardly distinguishable, see Fig. 8. From an autopilot design point of view, this of course is very encouraging since the winds were quite significant. Regarding the steep approaches, the second one was slightly steeper due to the somewhat higher initial altitude. The path tracking accuracy is confirmed by Fig. 9(a) (lateral path error during first helical approach) and Fig. 9(b) (altitude error during first helical approach). The time along the helix path has been stretched along the $\mathrm{x}$-axis while the deviations are represented on the $\mathrm{y}$-axis. The larger peaks at the beginning and the end are caused by switching to and from the spiral mode. Their quick reduction illustrates the good tracking capability of the autopilot. More details on the behaviour of the autopilot can be found in Ref. ${ }^{4}$.

The recorded thrust, speed, and altitude profiles for each test flight are depicted in Fig. 10. Obviously, the operating conditions along identical flight procedures are in close proximity hence the corresponding noise emission should be quite similar as well (note: constant aircraft configuration).

The behaviour of the autopilot regarding piloting technique, e. g. throttle activity, was received well by the pilots. During the maneuver, ride quality (load factors, intercepts, etc.) was of no concern.

\section{B. Measured noise levels}

The wind conditions at the only available test day have been adverse for flyover noise measurements. Wind gusts up to 15 knots have been recorded on the ground. At the airport winds between 15 and 20 kts with direction $\approx 255^{\circ}$ (runway heading $265^{\circ}$ ) were measured. At higher altitudes winds up to $28 \mathrm{kts}$ were encountered. Between and below clouds (broken cumulus, base $\approx 4000 \mathrm{ft}$, top $\approx 7000 \mathrm{ft}$ ) thermal turbulence was encountered. In the clouds, turbulence levels were considerably higher.

Weather requirements for aircraft noise certification e.g. would have been violated due to the wind on the test day ${ }^{\mathrm{g}}$. Therefore, the measured noise levels should be examined critically and should not directly be compared with data from other campaigns under the certified wind conditions. The weather and wind conditions did not change significantly during the 2 hours of the test flights hence the influence of wind on the measured data is assumed to be similar for all recorded flight events. This approximation is confirmed by only small variations in the measurements for identical approach procedures, i.e. agreement between level-time-histories for flights 4 to 6 (HeNAP), flights 1 and 7 (reference), and flights 2 and 3 (steep) respectively. Recorded time-level-histories of the a-weighted Sound Pressure Level [dBA] are depicted in Fig. 11 and 12. The depicted flight time was initiated for each test flight at a ground distance of $12 \mathrm{~km}$ to the runway threshold. Despite the adverse weather conditions, the recorded SPL timelines reveal very low background noise levels for all microfone locations of $55 \mathrm{dBA}$ down to $50 \mathrm{dBA}$ at some locations. The maximum sound pressure levels of the aircraft flyover events are therefore more then $20 \mathrm{~dB}$ above the ambient noise levels, which is the required margin for noise certification measurements. This supports the suitability of the measured noise data for comparing the noise impact among the tested approach procedures. At observer locations Mic1 to Mic6 the noise levels for all 7 test flights have been recorded. At the remaining locations only the HeNAP noise was perceived.

Noise levels at observer locations 5 and 6 are compared, to evaluate the potential noise reduction of the tested procedures along the common approach path. Noise level differences with respect to data of flight\%, presented in Tab. 3, indicate the anticipated noise relocation effects at Mic5 and Mic6 due to the spiraling and steep approaches.

All test flights pass the $I L S_{\text {mode }}$ marker, depicted in Fig. 2, with identical altitude, speed, and thrust setting. Therefore, observer locations 1 and 2, located in close proximity to this marker, are exposed to similar noise peaks along each flyover event, see Figs. 11(a) and 11(b). Fig. 13 shows the measured noise levels along the spiraling segments of flight 4 . The aircraft following the HeNAP is consecutively flying over Mic1, Mic8, and Mic7. Each of these individual flyover events can be identified in Fig. 13(a). Fig. 13(b) and 13(c) show the noise impact inside and outside off the helix along flight 4 . The approximate ground track distance from observers inside and outside of the helix are not constant since inner observers are closer to the ground track than the outer observers ${ }^{\mathrm{h}}$. The measured noise levels inside and outside of the helix do not significantly deviate from each other hence it can be concluded, that slightly more noise is emitted out

\footnotetext{
${ }^{\text {gICAO weather conditions }}{ }^{8}$, Annex 16, Appendix 2,2.2.2: temperature between 2 and $35^{\circ} \mathrm{C}$; no rain, dew, or snow; no ground-based temperature inversions; humidity between 20 and $95 \%$; wind conditions $10 \mathrm{~m}$ above ground: average wind $\leq 12 k t s$, average cross wind $\leq 7 k t s$, maximum wind $\leq 15 k t s$, max. cross wind $\leq 10 k t s$

h distance to flight ground track: Mic9 - $540 \mathrm{~m}$, Mic10 - 600m, Mic11 - $1060 \mathrm{~m}$, Mic12 - $880 \mathrm{~m}$
} 
off the helix. Overall, the maximum noise levels measured below the helical flight segments are comparable to noise levels recorded below the standard ILS approach path. Comparing the recorded noise levels for the reference approach at observer locations 3 and 5 (Figs. 11(c) and 11(e)) with measurements of the HeNAP at locations 7 and 8 (Figs. 12(a) and 12(b)), does not indicate additional ground noise increase due to the curved flight segments.

\section{Measured vs. predicted noise data}

Strong winds and wind gusts are known to distort the sound field in an uncontrolled manner hence complex noise shielding effects are corrupted. Therefore, the current data was not considered appropriate to validate the new DLR noise shielding code $\mathrm{SHADOW}^{5}$ hence the noise shielding effects were neglected in the comparison.

Recorded flight test data are provided as input for the computational noise prediction, whereas the prediction in section III is based on simulated input data. Noise prediction with PANAM ${ }^{1}$ is based on semi-empirical, parametric noise source models to quickly identify low-noise aircraft designs and noise abating flight trajectories. Major aircraft noise sources are modelled but interactions have to be neglected. The software was not developed to precisely predict absolute noise levels on the ground but to provide trends as early as possible in the preliminary aircraft design phase. Weather effects, e.g. wind, temperature gradients, and topographic effects, are not accounted for. Discrepancies between measurement and prediction are expected to increase with large distances between observer and source due to the implemented approximations for atmospheric sound propagation effectsi. Finally, only noise level differences between the tested procedures should be considered for the comparison of experiment and prediction.

Predicted max. SPL noise footprints for each test flight are shown in Fig 14 . The noise was predicted based on recorded data during the flight test. At a ground distance of $12 \mathrm{~km}$ to the runway threshold, computations were started and the reference time was set to zero. The noise computation for flight1 to flight6 has been stopped right before the approach was aborted to initiate the go-around. Observer locations are depicted with pink markers on the isocontours. The aircraft configuration was held constant during all test flights ${ }^{j}$, hence ground noise impact is mainly determined by altitude, speed, and thrust setting. The shape of the noise isocontours can be directly correlated with the recorded flight data, e. g. increased noise levels early along flight1 can be correlated to a thrust peak between 50 - $70 \mathrm{~s}$ of flight time depicted in Fig. 10(a). Furthermore, the maximum levels can be identified for each microphone in the corresponding time-level-history plot.

Fig. 15 shows the predicted vs. the measured time-level-history at microphones 1 to 6 located along the common approach path. The trends and level differences are in good agreement between computation and experiment, although major discrepancies are experienced for the steep approach procedure. At observer locations 3 to 6 the noise predictions are significantly lower than the experimental data. This is the case for both steep approaches; the dashed lines show results for the second steep approach. The effect is not related to aircraft configuration or operating condition changes since the corresponding input data show no irregularities. According to the thrust setting along both steep approaches, depicted in Figs. 10(b) and 10(c), the engine is running on very little RPM. Engine noise seems to be significantly underpredicted for idle operation of the ATTAS engines, see Fig. 16.

Prediction and experimental data at observer locations 7 to 12 are depicted in Fig. 17. The agreement of the trends and level differences are satisfying with existing discrepancies due to the aforementioned engine noise underprediction and adverse wind conditions.

Overall, predicted and measured noise level differences between individual test flights are in good agreement. Exemplary, the level differences between a HeNAP (flight4) and a reference approach (flight1) are presented. For the HeNAP the following noise reduction was confirmed by prediction and experiment: $\approx 10 \mathrm{dBA}$ at Mic3, $7-8 d B A$ at Mic4, and $\approx 8 d B A$ at both Mic5 and Mic6. Finally, it can be concluded that the applied noise prediction methods are capable of predicting the noise distribution along helical flight procedures. The requirements for a feasible comparative noise evaluation are fulfilled with the existing methods.

\footnotetext{
${ }^{\mathrm{i}}$ American National Standard Method for the Calculation of the Absorption of Sound by the Atmosphere, ANSI S1.26-1978, 1978

${ }^{\mathrm{j}}$ constant flap setting with deployed gear
} 


\section{ATC and flight operational aspects}

As already mentioned, the feasibility of integrating the HeNAP flight procedure into an existing background scenario should be first prepared and tested at regional airports with a small number of conventional aircraft movements or at times with low air traffic figures (e.g. night time where the noise aspect is of paramount importance). The Forschungsflughafen Braunschweig with its location, air traffic, flexibility, and experienced research personnel, i. e. DLR flight test crew and flight operation department offers splendid conditions for the initial evaluation of such new operational flight profiles. After the promising results in terms of sound pressure levels dislocation to ideally unpopulated areas for that kind of procedure as described in the sections before, this chapter leaves the perspective of the single event environmental assessment and addresses first subjective comments of an operational implementation of this specific noise reduction approach procedure into a complete air traffic system with multiple flights.

For this reason, the complete flight test campaign has been attended in the background by research staff from DFS directly from the tower building and the procedures have been kindly commented by the two tower controllers on duty ${ }^{\mathrm{k}}$. Their subjective statements regarding workload and situational awareness considering the various approaches are summarized below. A radar display based on the system PHOENIX was available for both controllers for the presentation of airborne aircraft positions around EDVE (range normally until flights reach FL150); a further monitor for the specific illustration of surface movements was not at hand. Meteorological parameters i.e. wind speed and direction, ambient pressure, temperature, and humidity were logged continuously from Forschungsflughafen Braunschweig around the airport ground during the seven approaches and delivered afterwards to the partners for further processing. There was visual contact between the control tower and the flying testbed ATTAS, which flew under instrumented flight rules (IFR), throughout all helical segments (VMC). Before commencing each individual approach procedure the corresponding tower controller issued a low approach clearance to the pilots for RWY 26 in coordination with the responsible area control center located in Bremen. Only for the last flight (flight'7, reference approach procedure) there was need for an ILS landing clearance on RWY 26, given 10 NM before touchdown.

With to regard to separation, the vertical separation between two aircraft has to be at least $1000 \mathrm{ft}$. As the helix initial altitude $h_{\text {init }}$ is set at $7500 \mathrm{ft}$ and $h_{\text {end }}$ at $550 \mathrm{ft}$, the aircraft descends about $2300 \mathrm{ft}$ in every spiral (altogether 3 turns). The length of a completed spiral segment is about 7 NM. Considering the relative constant flight speed of about $160 \mathrm{kts}$ during the HeNAP segments (Figs. 10(d) to 10(f)) results in a flight time of 150 seconds for one spiral element.

Horizontal separation minima ${ }^{11}$ according to Tab. 4 must be applied when both aircraft are landing at the same runway (in our case RWY 26). Radar separation minima between two aircraft are usually 3 NM below FL $195^{1}$.

However, as long as wake turbulence behaviour within the helical segments has not been further detailed, only one aircraft at a time should be allowed to enter and follow the HeNAP in its current design $\left(n_{\text {turn }}=3\right)$; which is a very conservative approach considering the before mentioned separation values ${ }^{\mathrm{m}}$.

In dedicated interviews the tower controllers have been asked to give statements after each approach procedure. Whereas they reported no specific handling and controlling challenges for reference and steep approach procedures, the defined HeNAP raised safety and efficiency questions in terms of missed approaches and blocking northbound departures and northern aerodrome traffic circuit for a long time. Therefore a negative influence on the throughput of an airport is estimated; however this task was not in the focus of this study and further analysis is thus necessary. In addition, subjective work load of the involved air traffic controllers increases as they have to watch carefully the altitude information of the ATTAS aircraft on a primarily two-dimensional display and be aware of the number of spiraling segments during HeNAP. Summarizing the comments, this kind of noise abatement procedure is not applicable during daytime with current operational state-of-technology at bigger airports and under adverse weather conditions.

These aspects have to be addressed and clarified in advance before transferring helical noise abatement flight tests to other airports which are confronted with more flight movements.

\footnotetext{
${ }^{\mathrm{k}}$ One tower runway and ground controller at one communication frequency and in addition one tower controller assistant for planning and coordinating issues. Note that the TWR EDVE is not operated by DFS.

${ }^{1}$ System PHOENIX

mATTAS VFW 614: wake turbulence classification MEDIUM
} 


\section{Summary and conclusion}

A new approach procedure has been investigated at DLR. Initial computational evaluation has predicted significant noise reduction potential for the so-called Helical Noise Abatement Procedure (HeNAP). High ground noise levels can be dislocated from the common ILS approach path and concentrated to the area along the helical flight segments. Obviously, this area is subject to multiple flyover events per approaching aircraft and hence increased noise pollution. But at the same time, ground noise levels along the entire preceding flight path are significantly reduced. Obviously, fuel demand, required flight time, and engine emissions along a helical approach are significantly increased compared to standard ILS approaches. A systematic operational and economical evaluation has to be performed in the future as this was not possible under the given circumstances.

In parallel to the computational activities, a DLR flight test was prepared to test a newly developed autopilot with improved weather capabilities. The flight test maneuvers were conducted in the form of landing approaches to investigate both the new autopilot and the HeNAP at the same time. For a comparative analysis of the HeNAP, a steep approach and a standard ILS approach were included in the test program. Due to the support of volunteers and the technical equipment provided by RWTH Aachen University, the flight tests could be combined with a dedicated ground noise measurement campaign. Ground noise levels have been recorded at selected observer locations along the flight ground track. Measurements confirm the anticipated noise dislocation effects along the spiraling approach. Compared with the reference approach, a noise reduction of approximately $8 \mathrm{dBA}$ was measured at observer locations 5 and 6 for the HeNAP.

The test flights have been simulated and noise levels were predicted according to recorded flight data. The predicted and measured noise level differences between individual test flights are in good agreement with the experimental data. It can be concluded, that the applied computational tools are able to predict noise distribution along helical flight procedures hence can be applied towards a comparative design of new lownoise flight trajectories.

The tower controllers reported no specific handling and controlling challenges for reference and steep approach procedures but the HeNAP raised safety and efficiency questions. Helical approaches increased the subjective work load of the tower controllers. A clear distinction between these first impressions and trends and a later evaluation phase with a detailed operational concept has to be made when interpreting the results of the paper. It can be summarized that this kind of procedure is not applicable during daytime with current state-of-technology or under adverse weather conditions. Helical approaches become more feasible for implementation at small, low-frequented regional airports or during night hours to avoid possible noise related curfews. Significant economical benefit due to extended operating hours could be achieved ${ }^{12}$.

Overall, the $2 \mathrm{hr}$. flight test led to an impressive amount of experiment data that covers flight data, noise measurements at 12 microphone stations on the ground, flight crew comments, and observations in the control tower. Although safety issues, e.g. engine failure and missed approaches, become crucial for these non-standard flight procedures they have not been in the scope of the presented work. Initial results with respect to safety during spiraling approaches have been discussed by Volovoi et al. in $2009^{13}$.

\section{Acknowledgments}

The authors would like to express their gratitude towards the ATTAS flight crew, especially Mr. Press ${ }^{n}$ and Mr. Leißling ${ }^{\circ}$, for their excellent job in preparing and operating the simulation and the flight test. Their willingness to conduct the autopilot flight test maneuver in the form of a landing approach allowed considerable additional value to be generated from the two hours flight test. The authors would like to thank Prof. Henke ${ }^{\mathrm{p}}$, Dr. Geyr von Schweppenburg ${ }^{\mathrm{q}}$, and Dr. Bals ${ }^{\mathrm{r}}$ for their support towards an add-on ground noise measurement campaign. Furthermore, the authors thank Prof. Henke for supporting the no-budget activities with the necessary acoustical equipment and additional manpower. The permission of local landlords around EDVE to install microphones on their property is greatly appreciated. The authors thank all involved volunteers of DLR and RWTH Aachen University for participating in this extracurricular activity.

\footnotetext{
${ }^{\mathrm{n}}$ DLR Flight Operations, Braunschweig

${ }^{\circ}$ Flight Dynamics and Simulation Group, Institute of Flight Systems, DLR

pHead of the Institute of Aeronautics and Astronautics, RWTH Aachen University

${ }^{\mathrm{q}}$ Head of the Department Configuration Design, Institute of Aerodynamics and Flow Technology, DLR

${ }^{\mathrm{r}}$ Head of the Department of System Dynamics and Control, Institute of Robotics and Mechatronics, DLR
} 


\section{References}

${ }^{1}$ L.Bertsch, G.Looye, T.Otten, M.Lummer: Integration and application of a tool chain for environmental analysis of aircraft flight trajectories, 9th AIAA Aviation Technology, Integration, and Operations Conference (ATIO) 2009, paper AIAA-2009-6954

${ }^{2}$ C.Hange, D.Eckenrod: Assessment of a C-17 Flight Test of an ESTOL Transport Landing Approach for Operational Viability, Pilot Perceptions and Workload, and Passenger Ride Acceptance, AIAA Aerospace Sciences Meeting and Exhibit 2007, paper AIAA-2007-1398

${ }^{3}$ J.R.Young, D.W.Hall: Executive Summary of Cal Poly/NASA Extreme Short Takeoff and Landing (ESTOL) Work, AeroTech Congress \& Exhibition 2005, paper SAE 200501-3177

${ }^{4}$ G.Looye: Flight testing of autopilot control laws: fly the helix!, German Aerospace Congress 2009, paper DLRK-2009121375

${ }^{5}$ M.Lummer: Maggi-Rubinowicz Diffraction Correction for Ray-Tracing Calculations of Engine Noise Shielding, 14th AIAA/CEAS Aeroacoustics Conference 2008, paper AIAA2008-3050

${ }^{6}$ L.Bertsch, W.Dobrzynski, S.Guérin: Tool development for Low-Noise Aircraft Design, 14th AIAA/CEAS Aeroacoustics Conference 2008, paper AIAA-2008-2995

${ }^{7}$ E.Anton, M.Basner, L.Bertsch., R.Henke: Assessment of a 3D Noise Abatement Procedure for Community Noise Impacts Based on Measurements and Simulations, submitted for presentation at the ICAS 2010 conference, September 2010
${ }^{8}$ International Civil Aviation Organization: International Standards and Recommended Practices, Environmental Protection, Annex 16 to the Convention on International Civil Aviation, Volume I, Aircraft Noise, 5. Edition, July 2008

${ }^{9}$ Dobrzynski, W.: Interferenzwirkungen durch Bodenreflexion bei Fluglaermmessungen an Propellerflugzeugen, DFVLR-Forschungsbericht, FB 81-28, 1981

${ }^{10}$ M. Pott-Pollenske, W. Dobrzynski, H. Buchholz, S. Guérin et al.: Airframe Noise Characteristics from Flyover Measurements and Predictions, 12th AIAA/CEAS Aeroacoustics Conference, 8 - 10 May 2006, Cambridge, Massachusetts, AIAA/CEAS Paper 2006-2567

${ }^{11}$ DFS: Manual of Operations for Air Traffic Control Services, Betriebsanweisung Flugverkehrskontrolle, Deutsche Flugsicherung GmbH, Langen, Germany, 22.11.2007, BA-FVK

${ }^{12}$ W.M.Wan Mohamed, R.Curran, F. van der Zwan, P.Roling: Modeling the Effect of Night Time Penalties on Commercial and Business Flights for Regional Airport Noise and Economics: Rotterdam Airport Case Study, 9th AIAA Aviation Technology, Integration, and Operations Conference (ATIO) 2009, paper AIAA-2009-7086

${ }^{13}$ V.Volovoi, G.C.Fraccone, A.E.Colon, M.Hedrick, R.Kelley: Agent-Based Simulation of Off-Nominal Conditions During a Spiral Descent (NextGen Vehicle NRA), 9th AIAA Aviation Technology, Integration, and Operations Conference (ATIO) 2009, paper AIAA-2009-7046

\section{Tables}

\begin{tabular}{|l|l|l|l|l|c|c|}
\hline procedure & $\begin{array}{l}60 \mathrm{dBA} \\
\text { isocontour } \\
\text { area }\left[\mathrm{km}^{2}\right]\end{array}$ & $\begin{array}{l}65 \mathrm{dBA} \\
\text { isocontour } \\
\text { area }\left[\mathrm{km}^{2}\right]\end{array}$ & $\begin{array}{l}70 \mathrm{dBA} \\
\text { isocontour } \\
\text { area }\left[\mathrm{km}^{2}\right]\end{array}$ & fuel $[\mathrm{kg}]$ & NOx $[\mathrm{kg}]$ & flight time $[\mathrm{s}]$ \\
\hline \hline Ref. approach & 78.05 & 41.66 & 7.01 & 208.56 & 1.71 & 450.5 \\
\hline HeNAP 2 & 199.67 & 55.01 & 26.08 & 299.05 & 2.34 & 675.5 \\
\hline HeNAP 3 & 102.63 & 39.03 & 12.77 & 329.08 & 2.37 & 833.0 \\
\hline HeNAP 4 & 67.73 & 29.74 & 14.60 & 363.21 & 2.50 & 971.5 \\
\hline
\end{tabular}

Table 1. Predicted environmental effects: HeNAP vs. reference approach

\begin{tabular}{|c|c|c|c|}
\hline observer & $\mathrm{x}[\mathrm{m}]$ & $\mathrm{y}[\mathrm{m}]$ & WGS coordinates \\
\hline Mic1 & -2924.32 & -21.55 & N 52.3222, E 10.6067 \\
\hline Mic2 & -3281.34 & -34.93 & N 52.3226, E 10.6119 \\
\hline Mic3 & -5210.96 & -64.57 & N 52.3245, E 10.6400 \\
\hline Mic4 & -5661.15 & -164.67 & N 52.3257, E 10.6465 \\
\hline Mic5 & -8239.39 & 6.07 & N 52.3263, E 10.6844 \\
\hline Mic6 & -8283.45 & -172.10 & N 52.3279, E 10.6848 \\
\hline Mic7 & -2796.36 & -4092.50 & $\mathrm{~N} 52.3586$, E 10.5994 \\
\hline Mic8 & -1713.22 & -3463.96 & N 52.3520, E 10.5844 \\
\hline Mic9 & -2355.22 & -3176.19 & $\mathrm{~N} 52.3500$, E 10.5942 \\
\hline Mic10 & -2995.14 & -3348.14 & N 52.3521, E 10.6033 \\
\hline Mic11 & -2425.86 & -4943.79 & N 52.3659, E 10.5929 \\
\hline Mic12 & -1750.79 & -4456.68 & $\mathrm{~N} 52.3610$, E 10.5836 \\
\hline helix center & -3200.0 & -2000.0 & N 52.3399, E 10.6079 \\
\hline RWY 26 & 0.0 & 0.0 & N 52.3196, E 10.5640 \\
\hline
\end{tabular}

\begin{tabular}{|l|l|l|l|}
\hline Flight & Type & $\Delta \mathrm{dB}$ Mic5 & $\Delta \mathrm{dB}$ Mic6 \\
\hline \hline flight2 & steep & -4.0 & -5.0 \\
\hline flight3 & steep & -4.5 & -6.0 \\
\hline flight4 & HeNAP & -7.5 & -8.5 \\
\hline flight5 & HeNAP & -7.5 & -7.0 \\
\hline flight6 & HeNAP & -6.5 & -9.0 \\
\hline flight7 & ref. & +1.5 & +1.0 \\
\hline
\end{tabular}

Table 3. Recorded max. SPL differences $\triangle$ dB w.r.t. flight1 at observer locations 5 and 6

\begin{tabular}{|l|l|l|}
\hline $\begin{array}{l}\text { Preceeding } \\
\text { aircraft }\end{array}$ & $\begin{array}{l}\text { Succeeding } \\
\text { aircraft }\end{array}$ & $\begin{array}{l}\text { Minimum } \\
\text { separation }\end{array}$ \\
\hline \hline HEAVY & MEDIUM & $4 \mathrm{NM}$ \\
\hline HEAVY & MEDIUM & $5 \mathrm{NM}$ \\
\hline HEAVY & LIGHT & $6 \mathrm{NM}$ \\
\hline MEDIUM & LIGHT & $5 \mathrm{NM}$ \\
\hline
\end{tabular}

Table 2. Observer coordinates

Table 4. Horizontal separation minima 


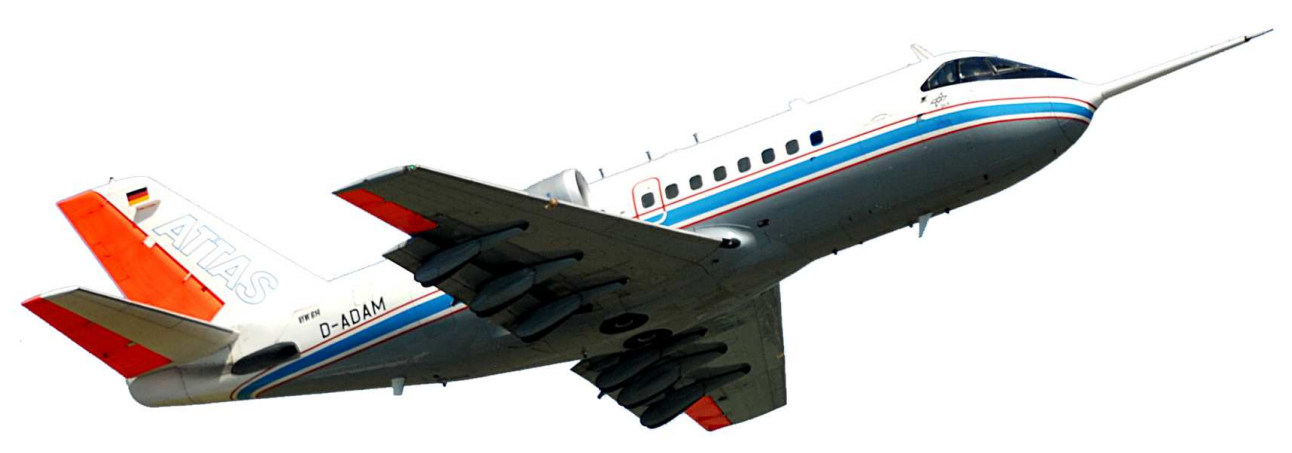

Figure 1. Flying test bed ATTAS of the German Aerospace Center

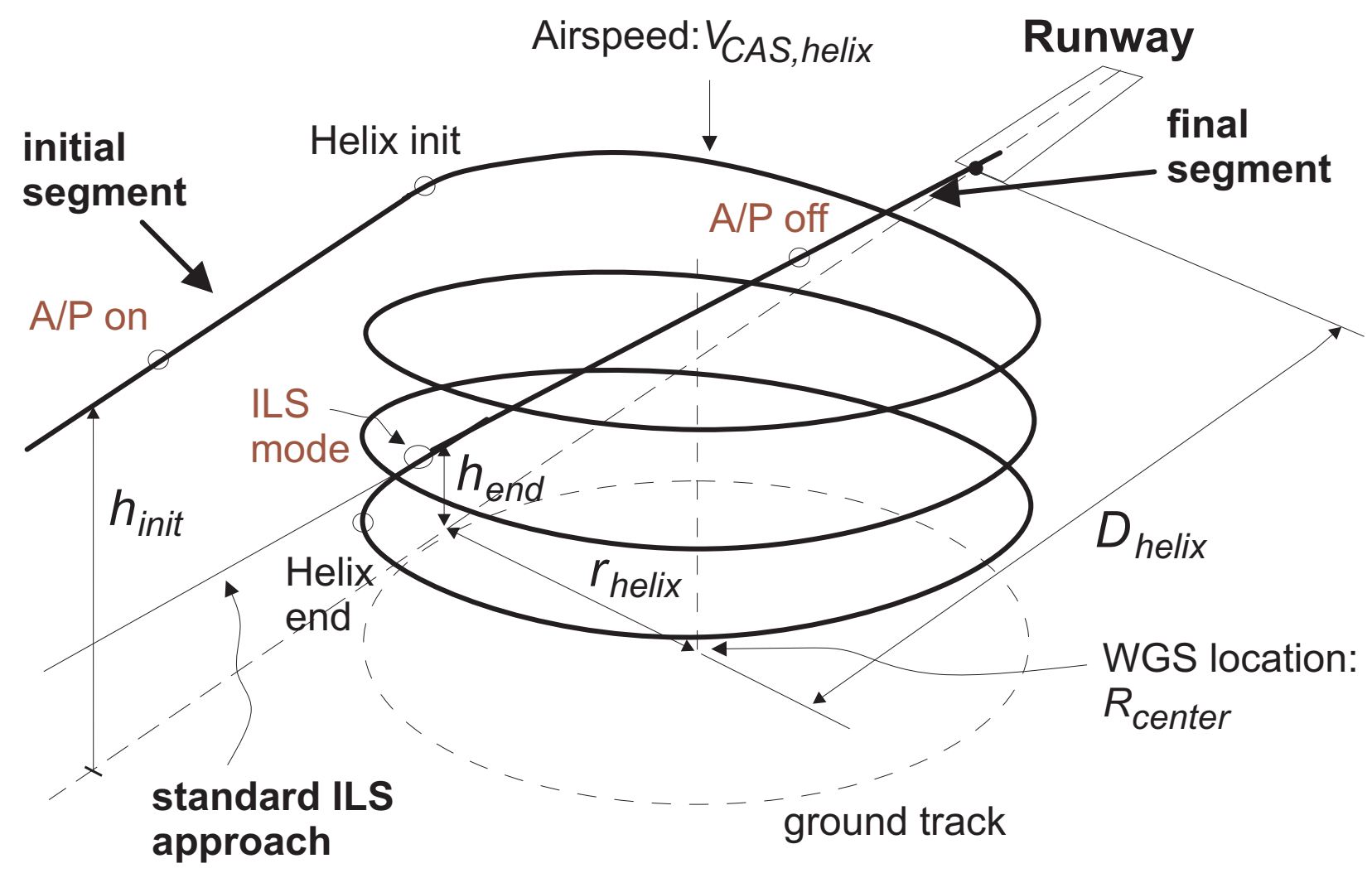

Figure 2. HeNAP: Parametric definition 


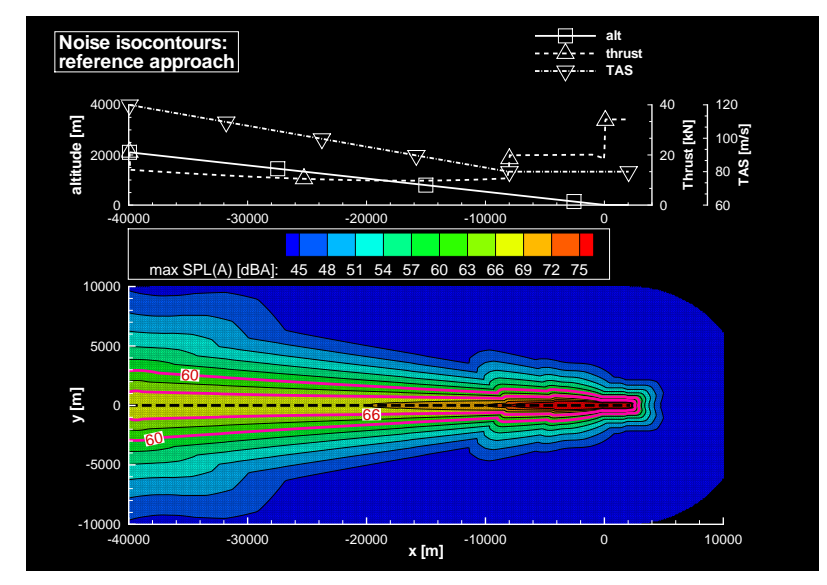

(a) reference approach

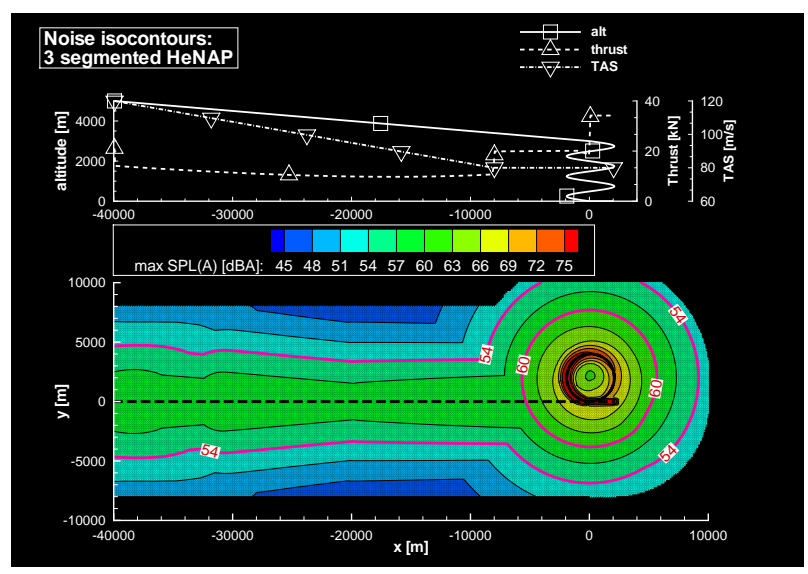

(c) HeNAP: 3 helical segments

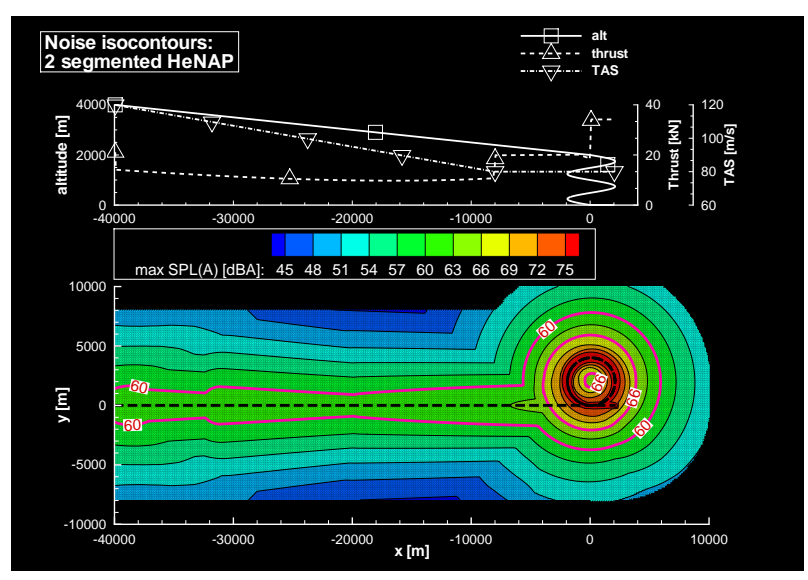

(b) HeNAP: 2 helical segments

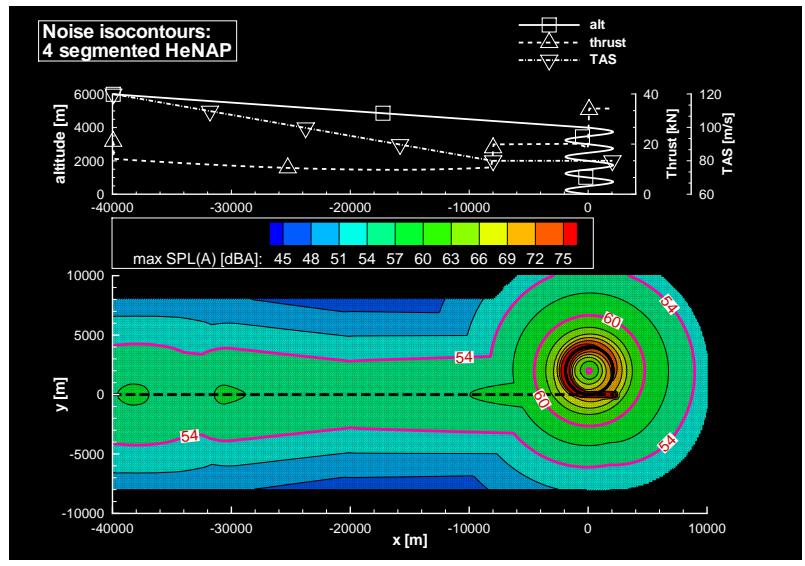

(d) HeNAP: 4 helical segments

Figure 3. Predicted max. SPL(A) isocontour plots for conventional, medium-range transport aircraft

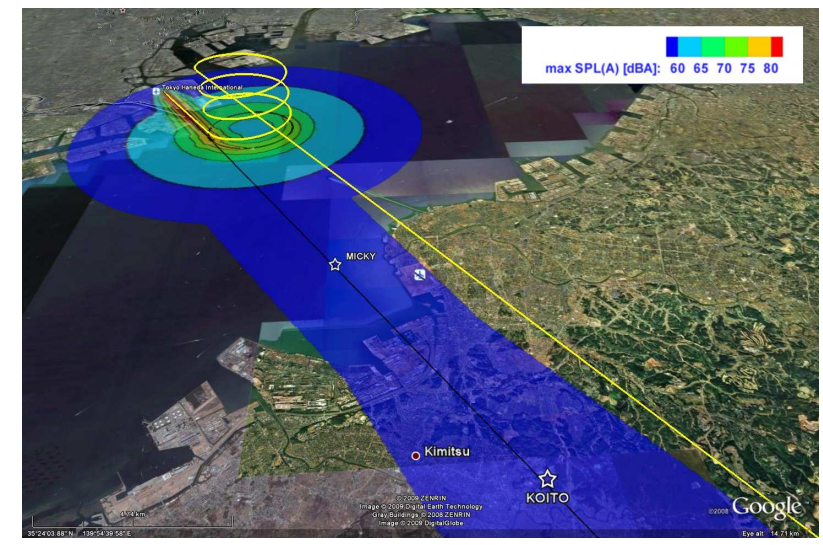

(a) 3 segmented HeNAP

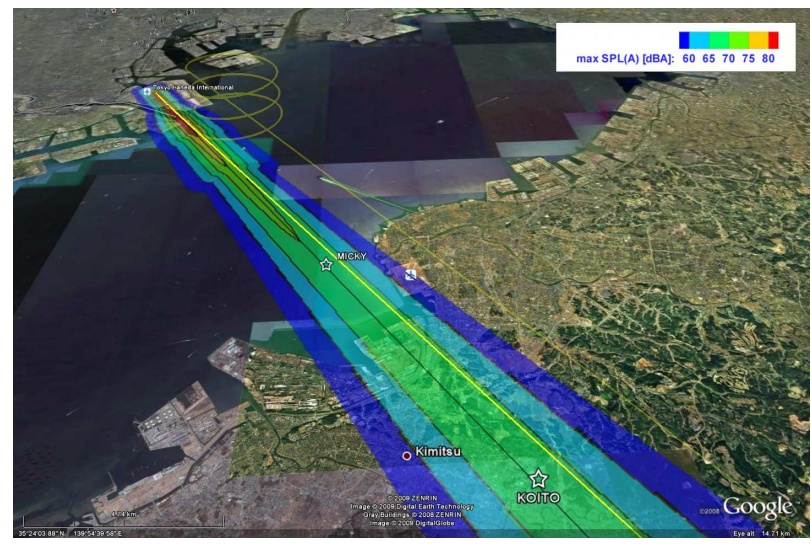

(b) Reference approach (Instrument Approach Chart - ILS Z OR LOC Z RWY34L)

Figure 4. Predicted max. SPL(A) isocontour plots: conventional, medium-range transport aircraft approaching Tokyo Haneda International Airport RWY 34 L (Google Earth) 

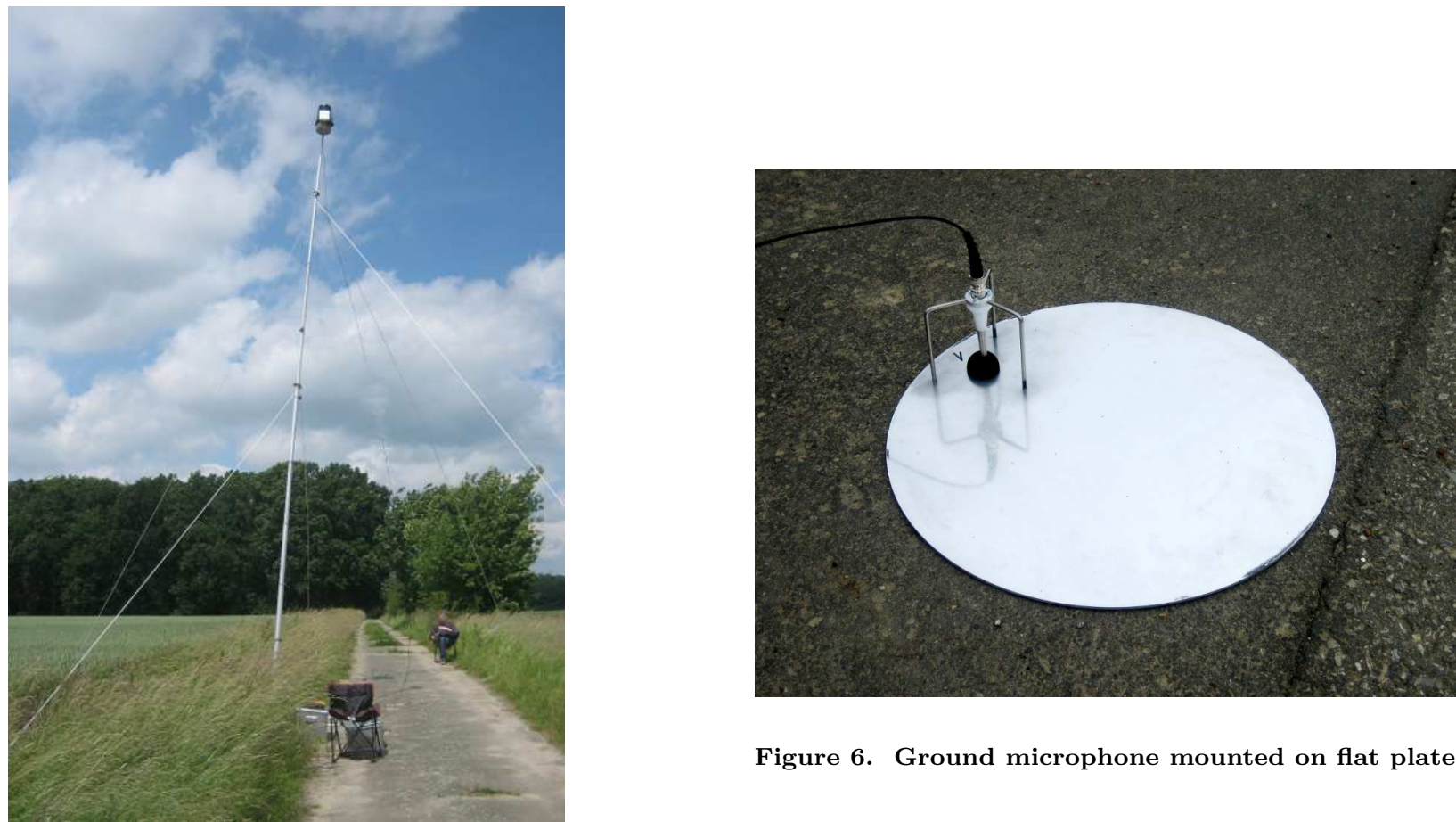

Figure 6. Ground microphone mounted on flat plate

Figure 5. Pole mounted weather sensor system

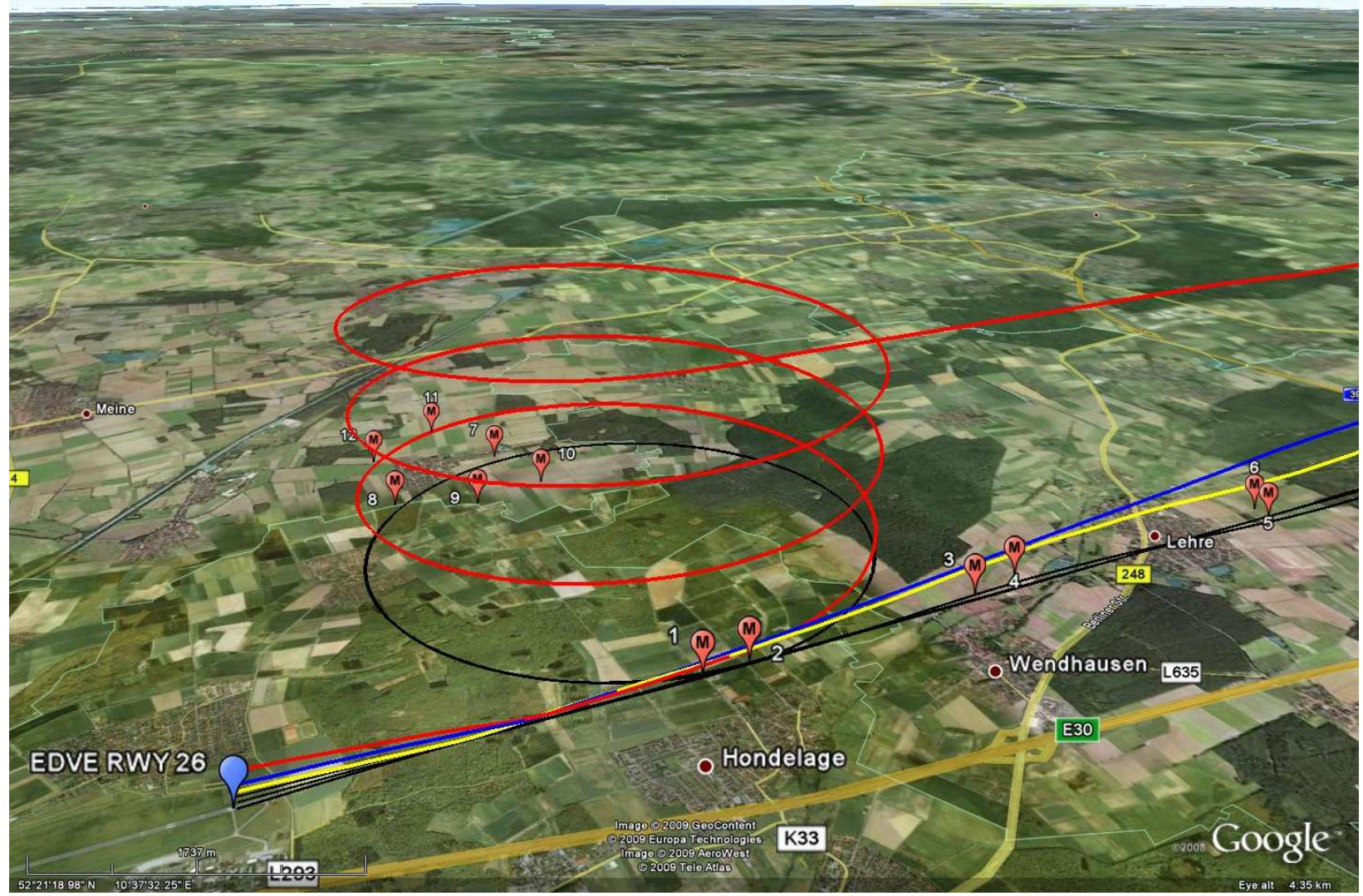

Figure 7. Flight ground tracks (black), flight paths (yellow: reference, blue: steep, red: HeNAP), and observer locations around EDVE (Google Earth) 


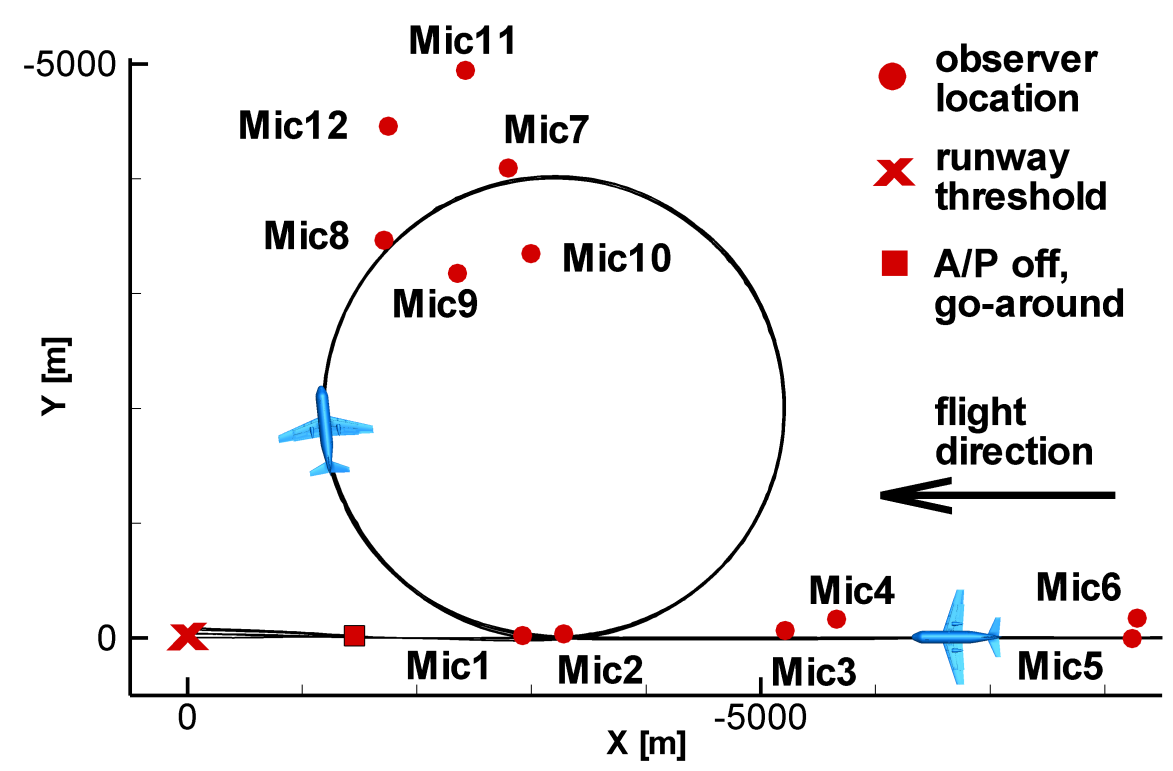

Figure 8. Observer locations along flight ground tracks (projection of the three HeNAP and two steep approach trajectories on the ground)

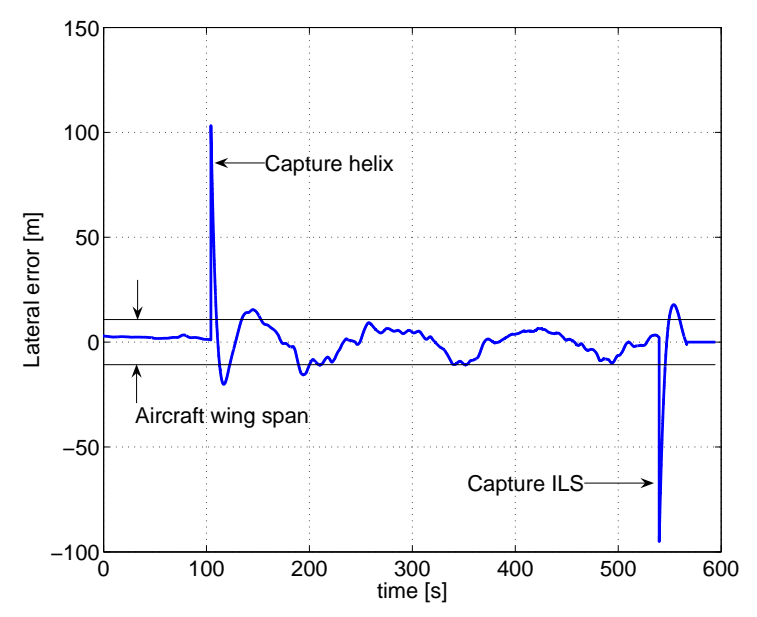

(a) Lateral path deviation during first helix approach

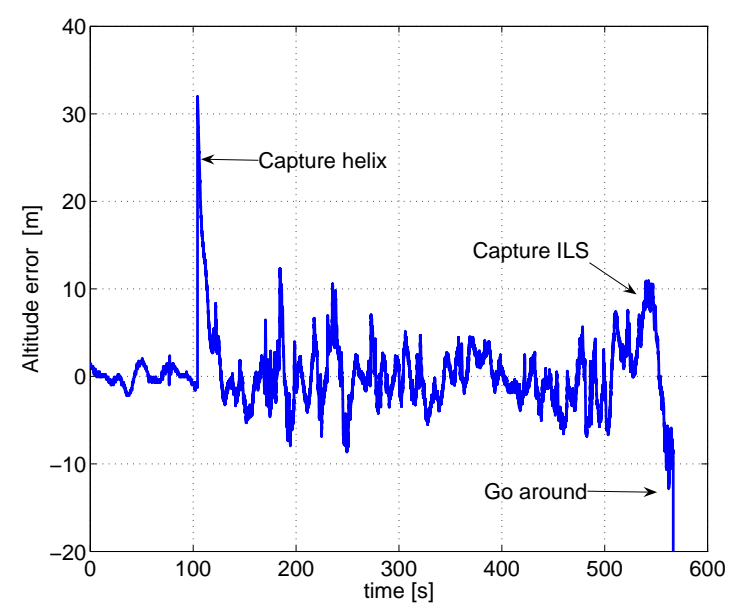

(b) Altitude error

Figure 9. Flight data visualisation 


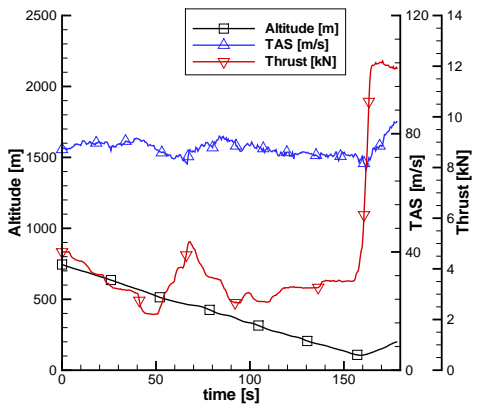

(a) flight1: reference

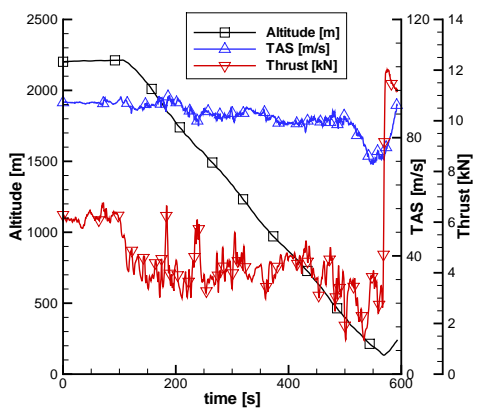

(d) flight4: HeNAP

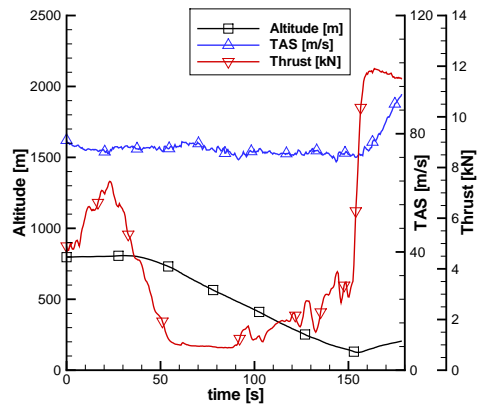

(b) flight2: steep

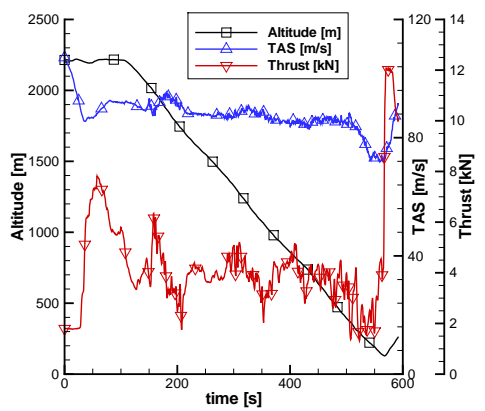

(e) flight5: HeNAP

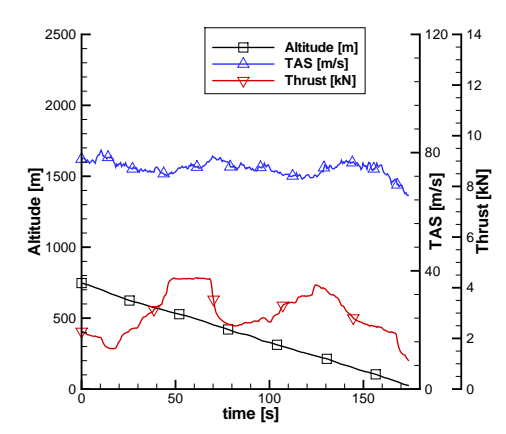

(g) flight7: reference

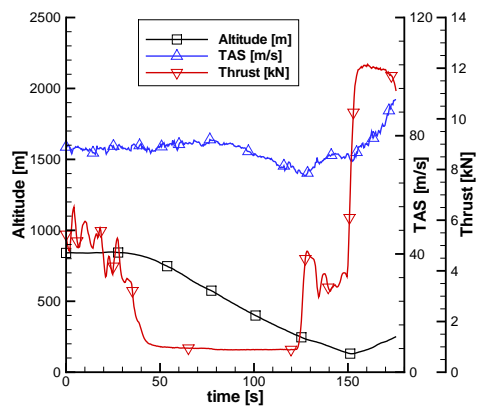

(c) flight3: steep

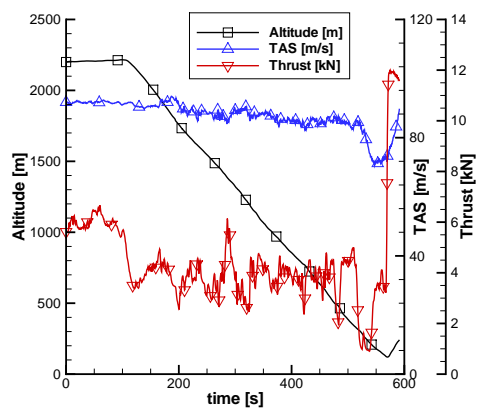

(f) flight6: HeNAP

(g) flightr: reference

Figure 10. Test flights 1-7: recorded thrust, TAS, and altitude history (constant configuration: gear deployed, flaps at $15^{\circ}$ ) 


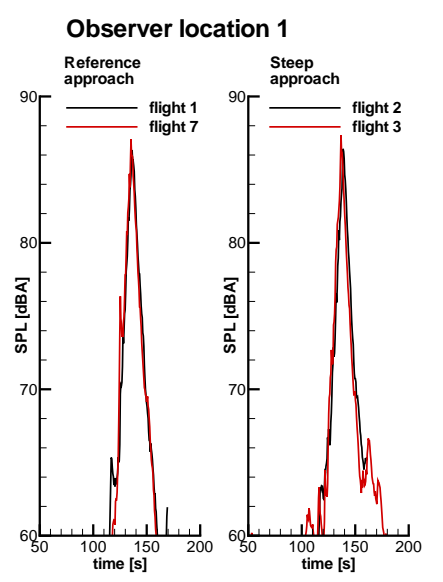

(a) Mic1

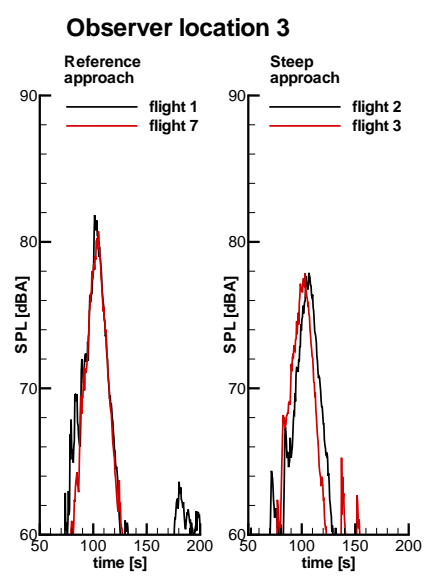

(c) Mic3

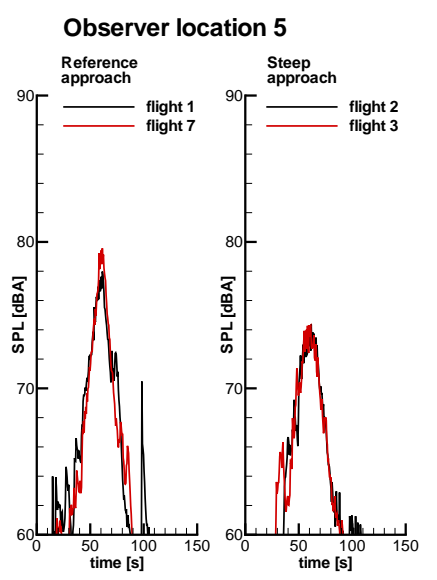

(e) Mic5
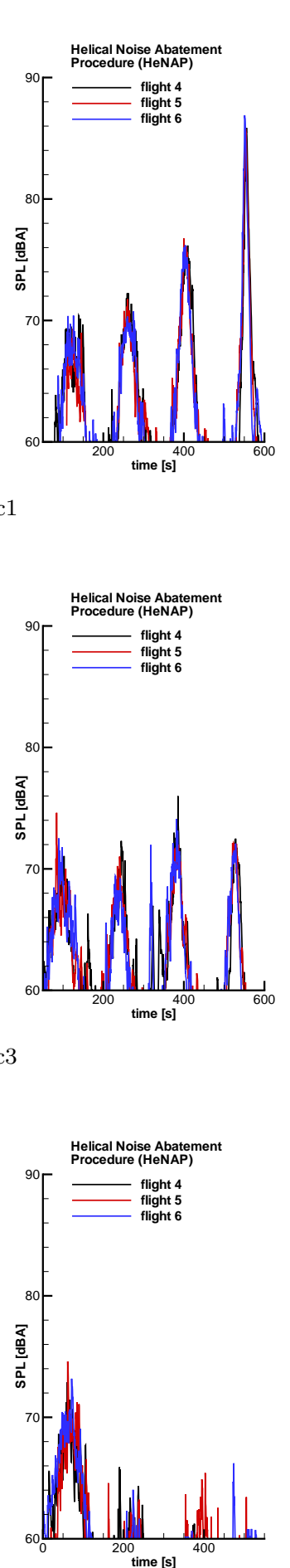

Figure 11. Measured SPL(A)-time-history at the observer locations 1-6

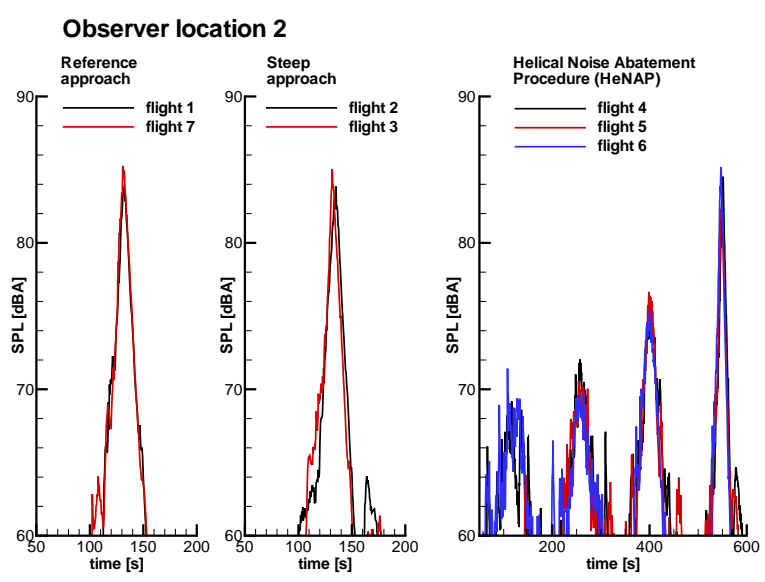

(b) Mic2

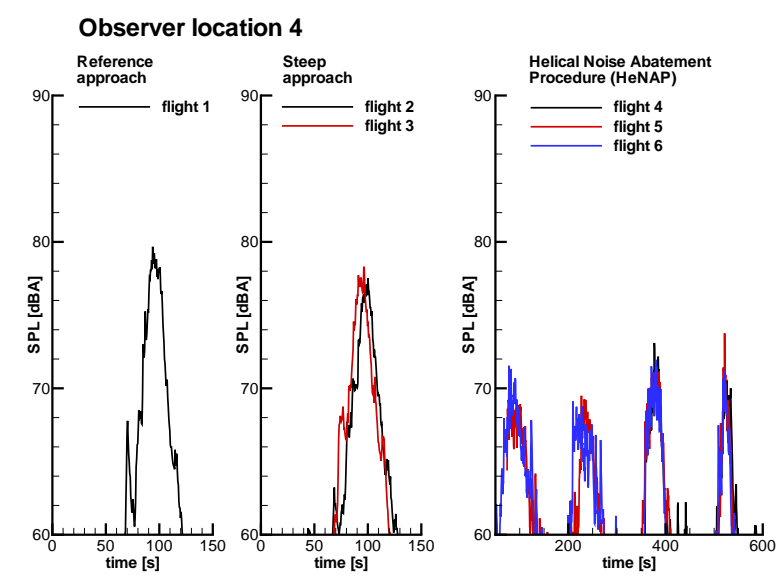

(d) Mic4

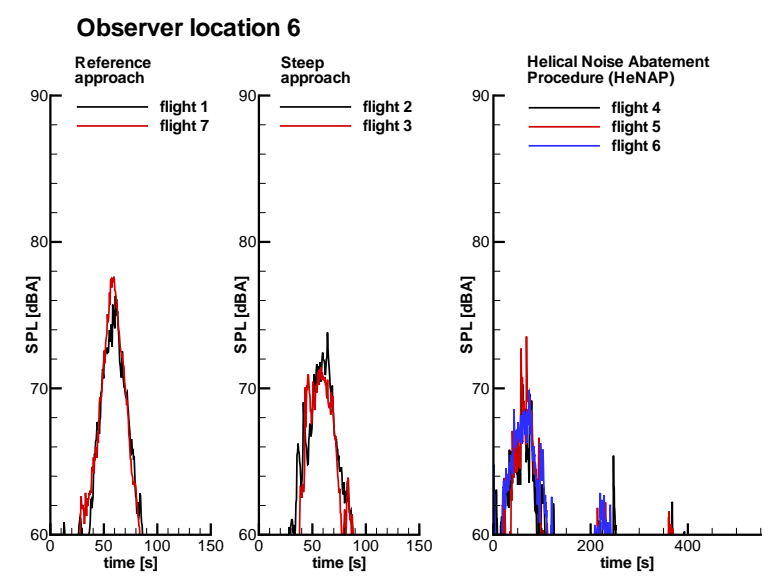

(f) Mic6 
Observer location 7

Helical Noise Abatement Procedure (HeNAP)

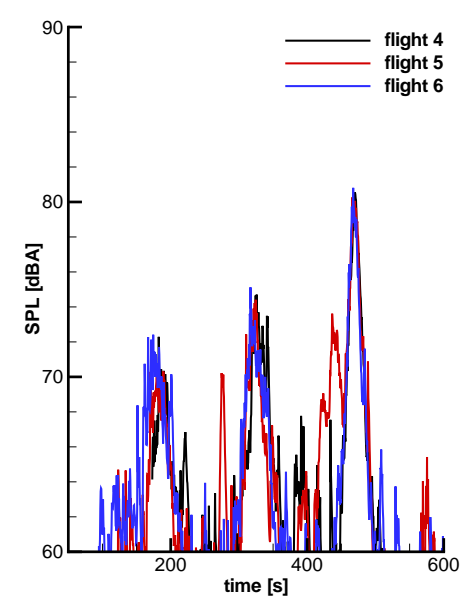

(a) Mic7

\section{Observer location 10}

Helical Noise Abatement Procedure (HeNAP)

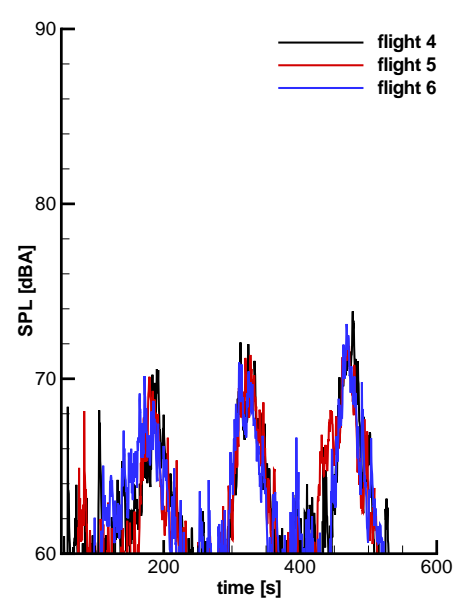

(d) Mic10
Observer location 8

Helical Noise Abatement Procedure (HeNAP)

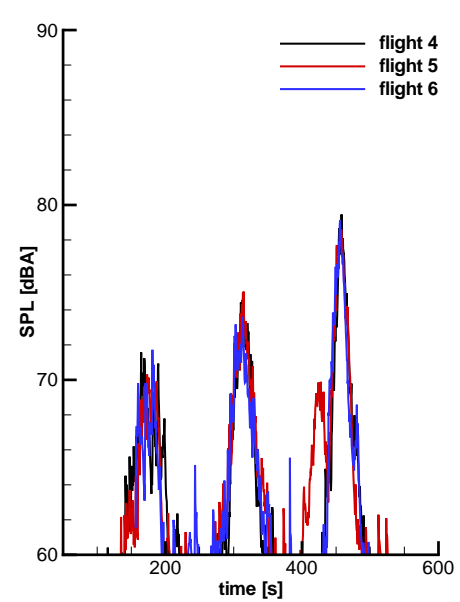

(b) Mic8

Observer location 11

Helical Noise Abatement Procedure (HeNAP)

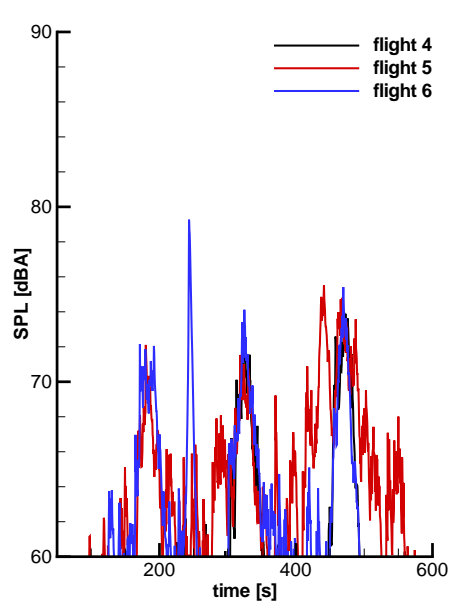

(e) Mic11

\section{Observer location 9}

Helical Noise Abatement Procedure (HeNAP)

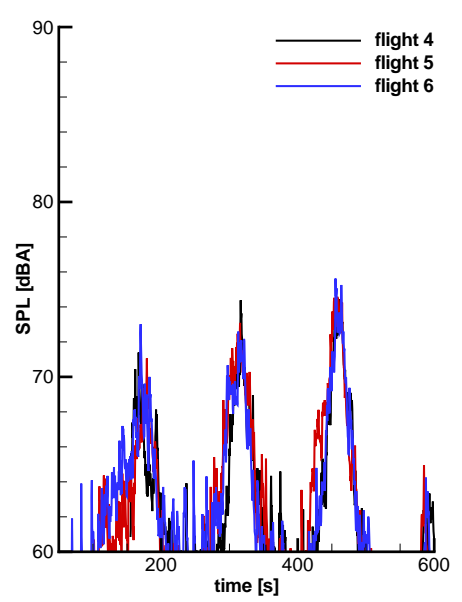

(c) Mic9

Observer location 12

Helical Noise Abatement Procedure (HeNAP)

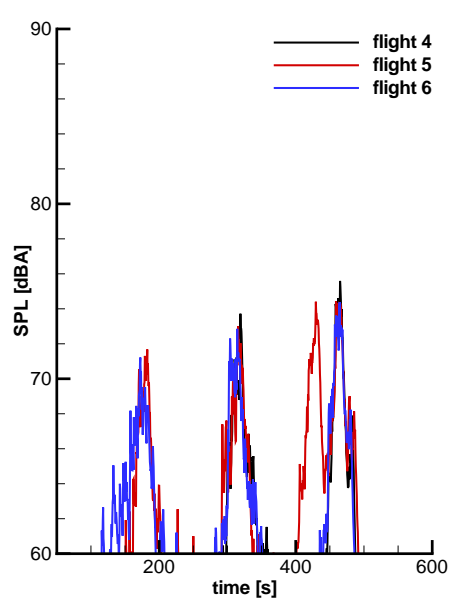

(f) Mic12

Figure 12. Measured SPL(A)-time-history at the observer locations 7-12

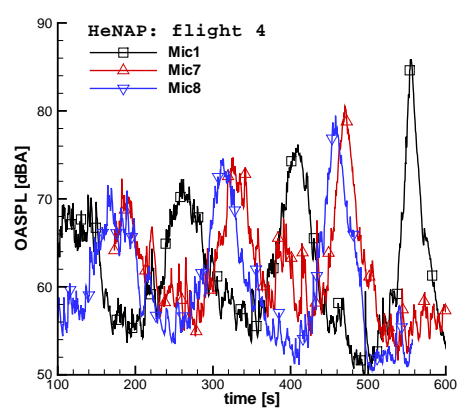

(a) Flyover of Mic1, Mic7, and Mic8

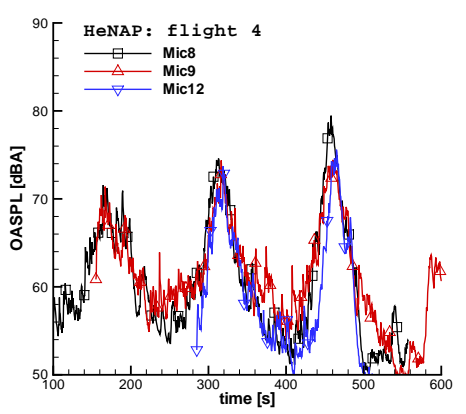

(b) Flyover of Mic8, Mic9, and Mic12

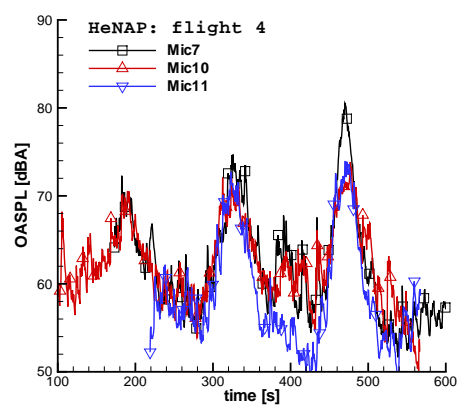

(c) Flyover of Mic7, Mic10, and Mic11

Figure 13. Flight 4: Measured SPL(A)-time-history at observers along the helical flight segment 


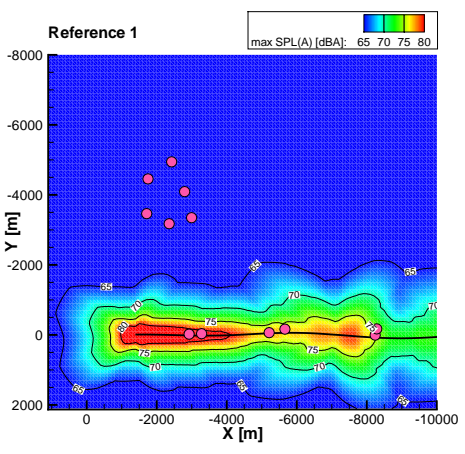

(a) flight1: reference

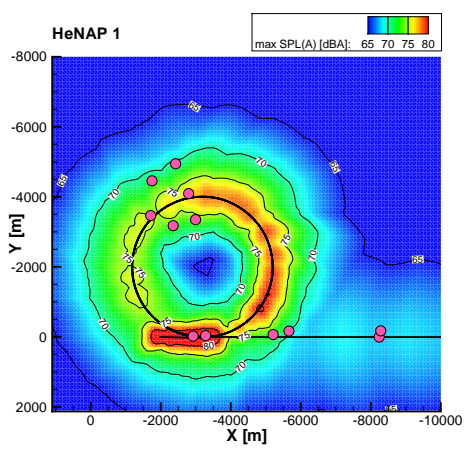

(d) flight4: HeNAP

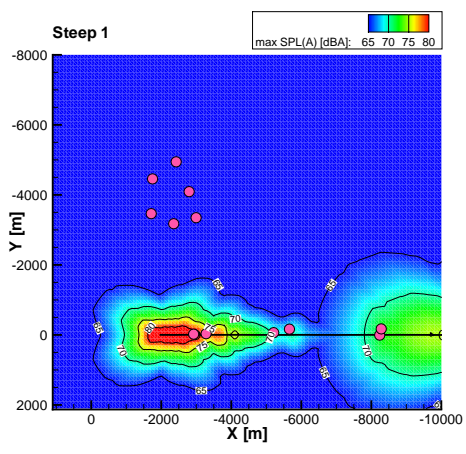

(b) flight2: steep

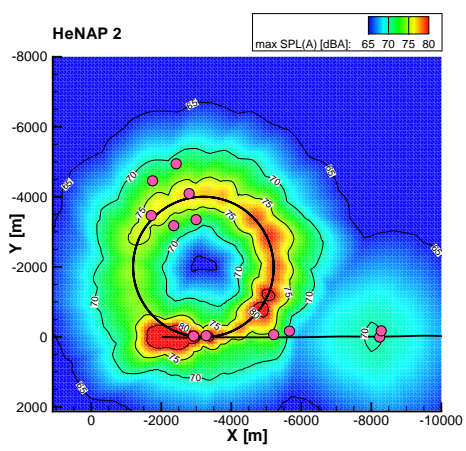

(e) flight5: HeNAP

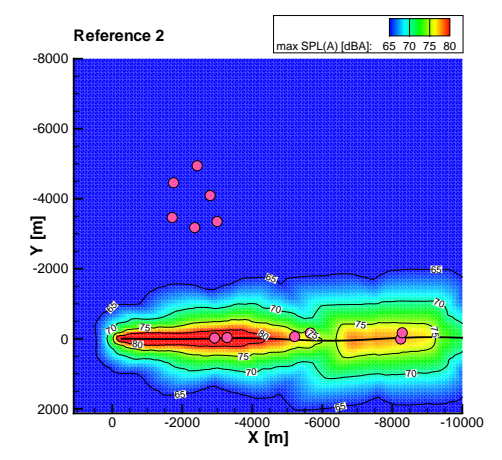

(g) flight7: reference

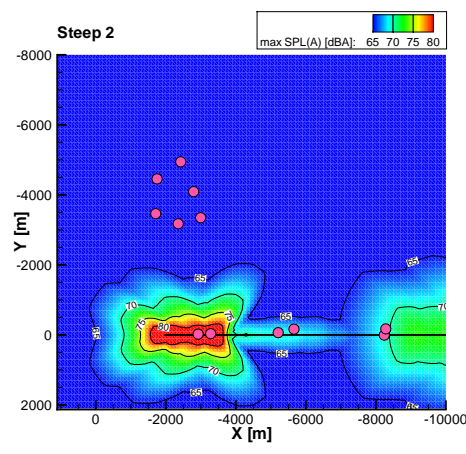

(c) flight3: steep

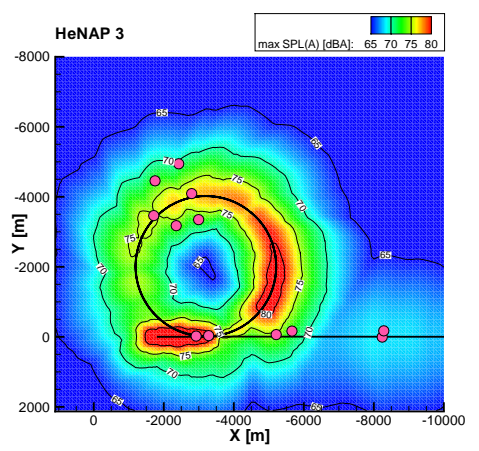

(f) flight6: HeNAP

(g) flightr: reference

Figure 14. Predicted SPLmax isocontours based on recorded flight data during test flights (ground microphones: pink markers, no shielding effects, $\sigma=100000 \frac{\mathrm{kN} \cdot \mathrm{s}}{\mathrm{m}^{4}}$ ) 
SPL time-history

microphone 1

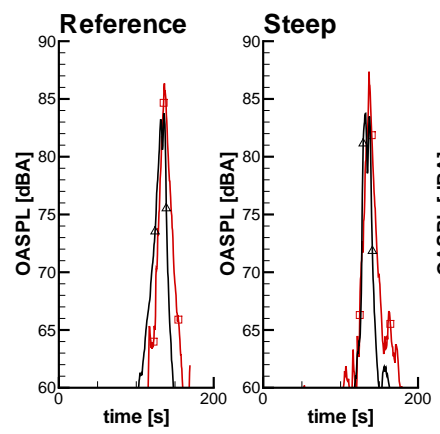

(a) Mic1

SPL time-history

microphone 3

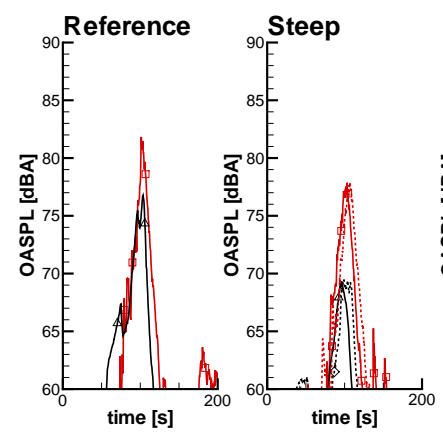

(c) Mic3

SPL time-history microphone 5

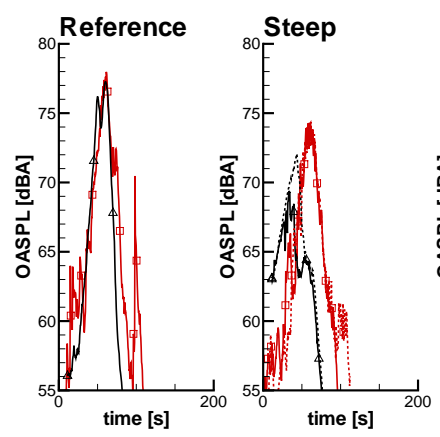

(e) Mic5
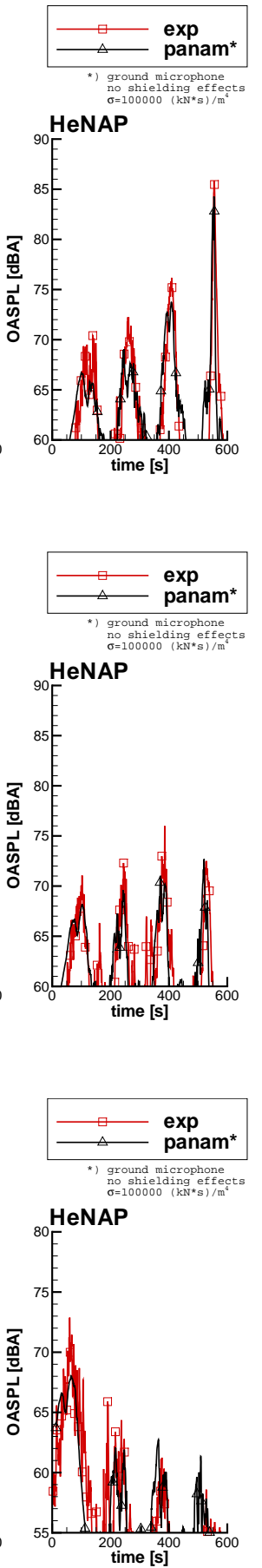

5 Figure 15. Flights 1, 3, and 4: Prediction vs. experimental data for observer locations along common approach
path 


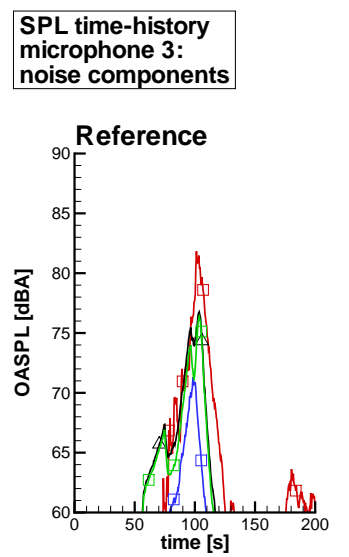

(a) Mic3
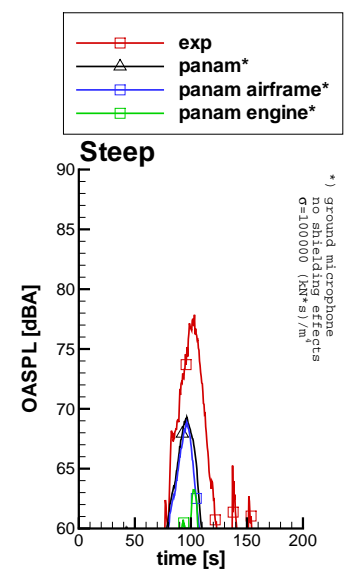
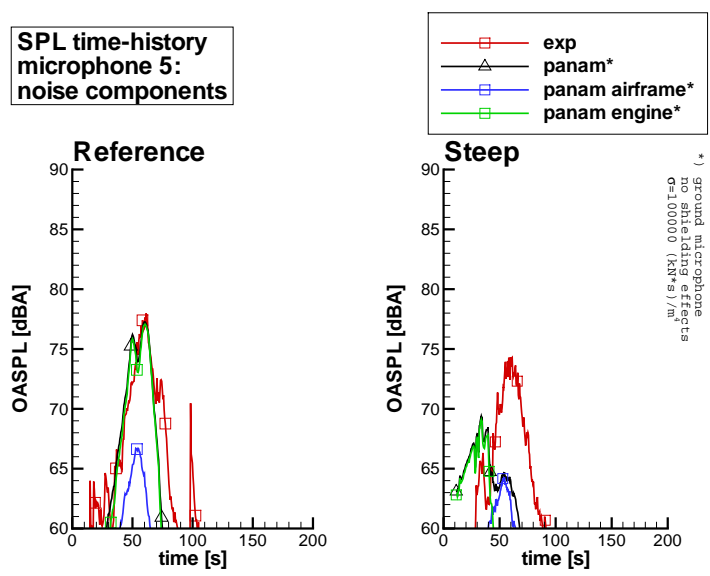

(b) Mic5

Figure 16. Flight 1 and 3: Predicted noise components vs. experimental data

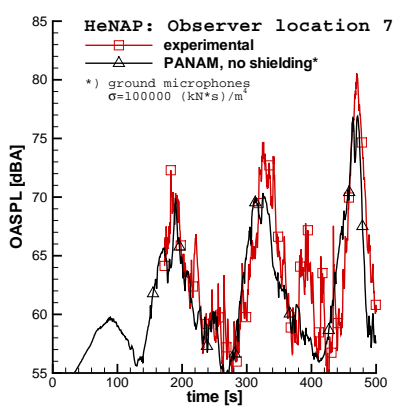

(a) Mic7

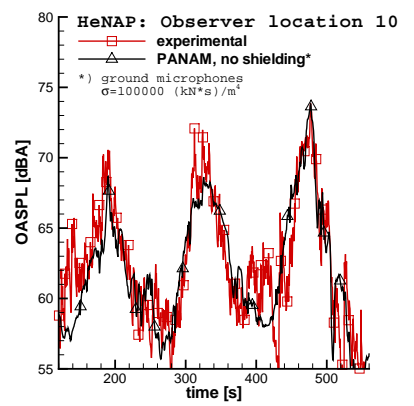

(d) Mic10

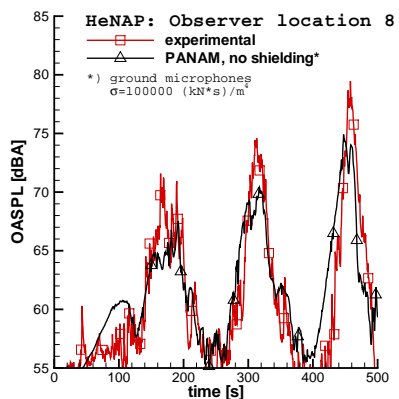

(b) Mic8

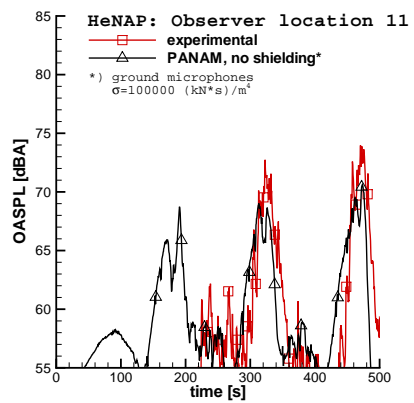

(e) Mic11

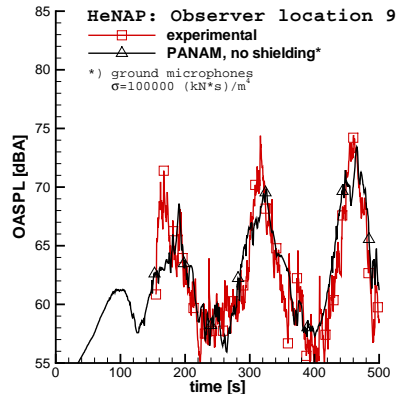

(c) Mic9

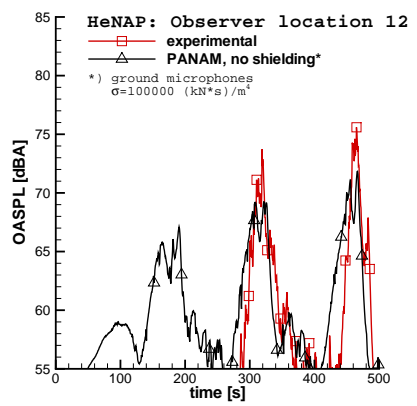

(f) Mic12

Figure 17. Flight 4: Prediction vs. experimental data for observer locations along helical flight segments 